\title{
A Fuzzy Logic-Based On-Demand Charging Algorithm for Wireless Rechargeable Sensor Networks With Multiple Chargers
}

\author{
Abhinav Tomar $\left.{ }^{(}\right)$, Member, IEEE, Lalatendu Muduli ${ }^{(}$, and Prasanta K. Jana ${ }^{\circledR}$, Senior Member, IEEE
}

\begin{abstract}
Mobile chargers have greatly promoted the wireless rechargeable sensor networks (WRSNs). While most recent works have focused on recharging the WRSNs in an on-demand fashion, little attention has been paid on joint consideration of multiple mobile chargers (MCs) and multi-node energy transfer for determining the charging schedule of energy-hungry nodes. Moreover, most of the schemes leave out the contemplation of multiple network attributes while making scheduling decisions and even they overlook the issue of ill-timed charging response to the nodes with uneven energy consumption rates. In this paper, we address the aforesaid issues together and propose a novel scheduling scheme for on-demand charging in WRSNs. We first present an efficient network partitioning method for distributing the MCs so as to evenly balance their workload. We next adopt the fuzzy logic which blends various network attributes for determining the charging schedule of the MCs. We also formulate an expression to determine the charging threshold for the nodes that vary depending on their energy consumption rate. Extensive simulations are conducted to demonstrate the effectiveness and competitiveness of our scheme. The comparison results reveal that the proposed scheme improves charging performance compared to the state-of-the-art schemes with respect to various performance metrics.
\end{abstract}

Index Terms-Fuzzy logic, wireless rechargeable sensor networks, mobile charger, on-demand, charging schedule

\section{INTRODUCTION}

W RELESS sensor networks (WSNs) have gained substantial research interest due to wide range of applications, including precision agriculture, habitat and environmental monitoring, IoT-based smart cities, industrial manufacturing, and so on [1]. However, energy constraint is the most critical issue as sensor nodes are small battery-powered that restricts their perpetual operation. Therefore, many energy saving [2], [3], [4] and energy harvesting schemes [5], [6] have been proposed. While the former schemes can only increase the operation time of a WSN to some extent, the latter ones are not always feasible as they supply energy from unpredictable environmental sources. The recent wireless energy transfer (WET) technology [7] and rechargeable lithium battery [8] have become very reliable to provide energy supply to the sensor nodes in a controllable and sustainable manner. As a result, wireless rechargeable sensor networks (WRSNs) have gained enormous research attention. In a WRSN, nodes are recharged by employing one or more mobile chargers (MCs) before their energy depletion and thus network can ideally work forever.

The MCs in a WRSN usually follow two basic schemes, periodic charging [9], [10] and on-demand charging [11], [12], [13]. In periodic charging, schedule of an MC is known

- The authors are with the Department of Computer Science E Engineering, Indian Institute of Technology (ISM), Dhanbad, Jharkhand 826004, India. E-mail: \{profession.abhinav, lalatendu.bapi123\}@gmail.com, prasantajana @yahoo.com.

Manuscript received 2 Sept. 2019; revised 23 Mar. 2020; accepted 21 Apr. 2020. Date of publication 0 . 0000; date of current version 0.0000.

(Corresponding author: Abhinav Tomar.)

Digital Object Identifier no. 10.1109/TMC.2020.2990419 a priori and the MC roams accordingly around the network 41 in a periodic fashion. However, due to heterogeneous and 42 dynamic energy consumption rate of the nodes, the fixed 43 charging schedule is not optimal. This leads to high node 44 failure rate and diminishes the charging performance. On 45 the contrary, an MC in on-demand charging can take real 46 time charging decision to fulfill energy demand of the sen- 47 sor nodes. This makes the on-demand scheme susceptible 48 to deal with high uncertainty in energy consumption of the 49 nodes. However, taking into account the battery deadlines 50 of requesting nodes, determining their efficient charging 51 schedule is very challenging and thus it is the most empha- 52 sized issue in the current research of WRSNs. Many on- 53 demand schemes have been developed till date. However, 54 most of them [11], [12], [13], [14], [15], [16] focus on the use 55 of a single MC to recharge only one sensor node at a time. 56 This can not be overlooked because a single MC with finite 57 power supply is not enough to recharge all the nodes, espe- 58 cially in dense WRSNs. At the same time, multi-node charg- 59 ing [17] of the nodes reduces waiting time incurred per 60 node, thereby improving charging efficiency. To this end, 61 this paper proposes an on-demand multi-node charging 62 scheme with multiple MCs to address the following funda- 63 mental issues: 1) How to distribute the multiple MCs in the 64 network without overlapping their charging regions so that 65 their charging workload are evenly balanced? 2) How to 66 ensure that a sensor node will not be recharged by more 67 than one MC at any time? 3) How to make wise and efficient 68 scheduling decisions by blending multiple network attrib- 69 utes, such as residual energy (RE), distance to MC (D), 70 energy consumption rate (ECR), and critical node density 71 


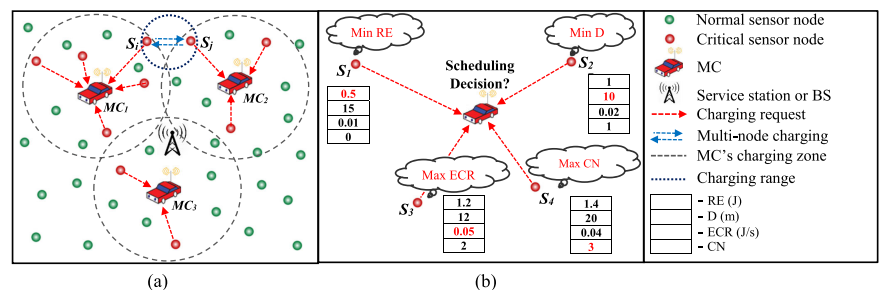

Fig. 1. System architecture showing an instance of charging scheduling problem: (a) Multi-node charging with multiple MCs. (b) Scheduling decision based on multiple network attributes.

72 (CN)? A system architecture addressing all such issues is 73 shown in Fig. 1. This can be noted from Fig. 1a that $M C_{1}$, $74 M C_{2}$, and $M C_{3}$ receive charging requests from 5,3 , and 2 75 nodes, respectively. This makes their charging load uneven 76 which leads to poor energy usage efficiency. This can also 77 be noted that the nodes $S_{i}$ and $S_{j}$ are within the charging 78 range of each other. As a result, both can be recharged by 79 either of both $M C_{1}$ and $M C_{2}$ through multi-node charging. 80 While taking the charging decision, this situation must be 81 avoided as radio interference caused by multiple MCs may 82 result in a lower energy transfer efficiency. In addition to 83 this, different network attributes may have different priori84 ties while scheduling the nodes. This is because the network 85 attributes RE, D, ECR, and CN are more critical for the 86 nodes $S_{1}, S_{2}, S_{3}$, and $S_{4}$, respectively, as shown in Fig. 1 b. 87 So, an effective and wise decision is necessitated by coordi88 nating different attributes to determine efficient charging schedule of the nodes.

This is noteworthy that there exist quite a few ondemand charging schemes [18], [19], [20], [21] which consider multi-node charging of the nodes with multiple MCs. The authors in [18], [19] formulated novel scheduling schemes for the longest delay minimization problem. They aimed at finding a closed charging tour for each $\mathrm{MC}$, while ensuring that a sensor node cannot be simultaneously charged by two or more MCs. However, in their approach, charging load among the MCs was uneven and optimization of other important performance metrics, such as average charging latency and energy usage efficiency was relaxed. Han et al. [20] proposed a charging scheme which first groups the nodes into uneven clusters and then determines the charging schedule for the MCs by considering both the traveling distance and residual lifetime of the nodes. Nonetheless, balancing the charging load among the MCs was missing which should not be overlooked. Similarly, the author in [21] proposed a charging scheme which clusters the energy demands of nodes to balance the charging load fairly between the MCs. This scheme minimizes the charging delay by maximizing the number of nodes recharged at each sojourn point. Still, this scheme lacks the joint consideration of various aforementioned attributes while making the scheduling decisions. As a result, many energy-hungry nodes may die immaturely. Furthermore, all such aforesaid schemes make their charging schedule by setting a fixed charging threshold (beyond of which sensor nodes send their charging request), which is not likely to cope with heterogeneous rate of energy consumption of the nodes. This makes the MCs perform unnecessary visits of energy-sufficient nodes which prolongs the waiting time of energy-hungry nodes and causes their energy starvation.
Inspired by the above facts, our proposed scheduling 122 scheme, which is based on fuzzy logic, incorporates follow- 123 ing novel and salient features to address the aforementioned 124 issues. First, the charging workload among the MCs is evenly 125 distributed so as to ensure the charging fairness. Second, the 126 Fuzzy Inference System (FIS) contemplates the aforemen- 127 tioned network attributes (i.e., RE, D, ECR, and CN) as inputs 128 to make the scheduling decisions based on the pre-set fuzzy 129 IF-THEN rules. Third, charging threshold for the nodes is 130 dynamically set up in order to avoid untimely charging 131 response by the MCs and thus improves the charging perfor- 132 mance. Note that fuzzy logic system is a powerful tool for 133 designing a charging scheduling scheme. Because, (i) it can 134 integrate multiple parameters in a balanced manner to make 135 real time decisions, even with incomplete and uncertain net- 136 work information [22], and (ii) parameters used for schedul- 137 ing decisions such as "lower residual energy", "closer 138 distance", etc., make their fuzzy representation easy and 139 realistic. To the best knowledge so far, we are the first to uti- 140 lize the fuzzy logic for on-demand charging of the nodes 141 using multiple MCs. We call our scheme the Fuzzy Logic- 142 based Charging Schedule Determination (FLCSD). The con- 143 tributions of this paper are as follows:

- Considering multiple energy constrained MCs, we 145 first present a novel partitioning approach to distrib- 146 ute the MCs in the network such that the charging 147 workload between them is evenly balanced.

- Then, an on-demand charging scheme is designed 149 which utilizes the fuzzy logic for determining the 150 charging schedule of the nodes by contemplating 151 various network attributes.

152

- A formulation of calculating an adaptive threshold 153 for the charging request is also presented with the 154 aim of timely recharging the nodes with uneven and 155 dynamic energy consumption rates.

- $\quad$ The proposed scheme is evaluated through extensive 157 simulations and compared with two existing and 158 similar works [20] and [21] in terms of energy usage 159 efficiency, average charging latency and survival 160 rate. The results are also statistically validated 161 through analysis of variance (ANOVA) test.

The rest of this paper is organized as follows. Section 2 pre- 163 sents a brief literature review of on-demand charging schemes. 164 Section 3 introduces some preliminaries, including the over- 165 view of fuzzy logic and system description. In Section 4, the 166 proposed FLCSD scheme is discussed in detail. Section 5 deri- 167 ves the simulation results followed by performance evalua- 168 tions. Finally, the paper concludes in Section 6.

\section{Literature ReView}

A plethora of research efforts have been devoted for devel- 171 oping the scheduling schemes in on-demand WRSNs. We 172 briefly review some of them as follows.

The Nearest-Job-Next with Preemption (NJNP) scheme 174 was proposed in [12] where the closest node from the MC is 175 selected to be charged next. However, in case of new charg- 176 ing request from a more closer node, the $\mathrm{MC}$ is preempted. 177 In case of dense networks, too much preemption of the MC 178 causes premature death of the nodes far away from the MC, 179 thereby making NJNP deficient. In [14], a charging scheme 180 
was proposed by taking into account both the requesting time of nodes and their distance from the MC, but in case of high number of charging requests occurred at the same time, it works same as NJNP which is not preferable. The authors in [23], aimed to schedule multiple MCs for energy supply to the nodes so as to maximize the covering utility. However, they failed to consider the other objectives, such as maximization of the survival rate along with minimum charging latency. In [11], the researchers focused to develop a metaheuristic-based charging scheme for scheduling the nodes. However, these schemes did not consider the multi-node charging of the nodes, thereby reducing the energy usage efficiency. Liang et al. [24] minimized the number of MCs for recharging the nodes and presented an approximation algorithm for the same. The authors in [16], came up with an approximated solution by utilizing the concept of smallest enclosing disk and determined optimal sojourn points for the MC. The authors in [13] designed a temporal-spatial charging scheme in order to plan an optimal charging path with the goal of minimizing the node deaths. Nonetheless, the above discussed schemes fail to take into account the multi-node charging and heterogeneous energy consumption of the nodes which should be given further consideration since it reduces the energy usage efficiency. The authors in [25] addressed the charging scheduling problem by considering both temporal deadline and spatial closeness among the charging requests. $\mathrm{Xu}$ et al. [26] solved the charging scheduling problem with multiple MCs by minimizing their total traveling distance. However, they failed to evenly balance the workload between the MCs, thereby causing more node deaths. Ma et al. [27] proposed a framework for multi-node charging using a single $\mathrm{MC}$ that measures the charging utility gain of each charged sensor, subject to the energy capacity of the MC. Gharaei et al. [28] proposed a route optimization of wireless mobile charger (ROWMC) algorithm in which two MCs are used, one for balancing the remaining lifetime of nodes and the other for guarantying that the energy level of nodes does not fall below a threshold.

Recently, the authors in [18] and [19] have reported algorithms for finding the charging tours of given MCs, subject to that no sensor can be charged by more than one $\mathrm{MC}$ at any time. Mo et al. [29] have addressed the multiple MCs coordination problem with an aim to minimize the total energy consumption of MCs by adjusting their moving and charging time. Khelladi et al. [30] considered multi-node charging for energy supply to the demanding nodes but they did not serve the objective of minimizing the charging latency. Moreover, considering multiple network attributes for taking scheduling decisions of the nodes was also missing. The author in [21] used the mechanisms of [30] and proposed a charging scheme which first clusters the energy demands to efficiently balance the charging load between MCs and then charges the nodes following certain scheduling rules. Finally, it computes the charging schedule for each MC by applying Traveling Salesman Problem (TSP) algorithm. The scheme aimed to improve charging performance in terms of charging latency and node failures. Han et al. [20] proposed the uneven cluster-based mobile charging (UCMC) scheme which first groups the nodes into uneven clusters and then determines the charging schedule
TABLE 1

Summary of the Existing on-Demand Charging Schemes

\begin{tabular}{lccccc}
\hline Paper Scheduling based & $\begin{array}{c}\text { Multiple } \\
\text { on multiple } \\
\text { network attributes }\end{array}$ & MCs & $\begin{array}{c}\text { Multi- } \\
\text { node } \\
\text { charging }\end{array}$ & $\begin{array}{c}\text { Charging } \\
\text { workload } \\
\text { balancing } \\
\text { between MCs }\end{array}$ & $\begin{array}{c}\text { Adaptive } \\
\text { charging } \\
\text { threshold }\end{array}$ \\
\hline [18] & No & Yes & Yes & No & No \\
{$[19]$} & No & Yes & Yes & No & No \\
{$[20]$} & No & Yes & Yes & Yes & No \\
{$[21]$} & No & Yes & Yes & No & No \\
{$[23]$} & No & Yes & No & No & No \\
{$[24]$} & No & Yes & No & No & No \\
{$[26]$} & No & Yes & No & No & No \\
{$[27]$} & No & No & Yes & No & No \\
{$[29]$} & No & Yes & No & No & No \\
{$[30]$} & No & No & Yes & No & Yes \\
{$[31]$} & Yes & No & Yes & No & No \\
Our & Yes & Yes & Yes & Yes & Yes \\
\hline
\end{tabular}

for the MCs by considering both the traveling distance and 242 residual lifetime of the nodes. However, multi-hop energy 243 transmission in their scheme suffers from low charging effi- 244 ciency. Recently, Tomar et al. [31] have proposed a fuzzy 245 logic-based charging scheduling scheme, but for a single 246 $\mathrm{MC}$ and thus it is infeasible for dense WRSNs. Note that the 247 aforesaid schemes [13], [20], [21], [24], [26], [29], [31] con- 248 sider uniform charging threshold for the nodes which is not 249 feasible in dynamic networks because the rate of nodes' 250 energy depletion vary dynamically with the time.

251

These limitations arise the need to further investigate the 252 scheduling problem of the nodes. The comparisons between 253 the proposed and the existing schemes with respect to five 254 key attributes are presented in Table 1.

\section{Preliminaries}

\subsection{Overview of Fuzzy Logic}

A fuzzy logic system has three functional units: fuzzification, 258 fuzzy logic controller, and defuzzification. In fuzzification, the 259 inputs in the crisp form are mapped to their fuzzy form 260 using some widely used membership functions. The fuzzy 261 logic controller performs decision making based on FIS and 262 rule base (composed of "IF\{set of inputs\}-THEN\{outputs\}" 263 linguistic statements). A fuzzy rule $R_{i}$ with $m$-inputs and 1- 264 output is expressed as follows:

$$
\begin{gathered}
R_{i}: \mathbf{I F}\left(I_{1} \text { is } A_{i 1} \text { AND } I_{2} \text { is } A_{i 2}\right. \text { AND ... AND } \\
\left.I_{m} \text { is } A_{i m}\right) \text { THEN }\left(O \text { is } B_{i}\right),
\end{gathered}
$$

where $\left\{I_{1}, I_{2}, \ldots, I_{m}\right\}$ denote the input linguistic variables 268 and $O$ is the output linguistic variable. Here, linguistic val- 269 ues for input and output variables are represented by $\left\{A_{i 1}, 270\right.$ $\left.A_{i 2}, \ldots, A_{i m}\right\}$ and $B_{i}$, respectively.

For evaluating the fuzzy outputs, the FIS uses some 272 membership function $(\mu)$ for reasoning. For example, the 273 fuzzy output for rule $R_{i}$ is evaluated (using $\mu$ and Eq. (1)) 274 as follows:

$$
\begin{gathered}
\operatorname{eval}\left(R_{i}\right):\left(\mu_{A_{i 1}}\left(I_{1}\right) \operatorname{AND} \mu_{A_{i 2}}\left(I_{2}\right) \text { AND } \ldots\right. \text { AND } \\
\left.\mu_{A_{i m}}\left(I_{m}\right)\right) \longrightarrow \mu_{B_{i}}(O) .
\end{gathered}
$$


Note that in fuzzy logic controller, conventional T-operators, such as MAX, MIN and Complement are used instead of combination operators, such as OR, AND, and NOT, respectively, and are expressed as follows:

$$
\begin{gathered}
\mu_{A_{i}}\left(I_{i}\right) \operatorname{AND} \mu_{A_{j}}\left(I_{j}\right)=\mu_{A_{i} \cap A_{j}}\left(I_{i j}\right)=\operatorname{MiN}\left(\mu_{A_{i}}\left(I_{i}\right), \mu_{A_{j}}\left(I_{j}\right)\right) \\
\mu_{A_{i}}\left(I_{i}\right) \operatorname{OR} \mu_{A_{j}}\left(I_{j}\right)=\mu_{A_{i} \cup A_{j}}\left(I_{i j}\right)=\operatorname{MAX}\left(\mu_{A_{i}}\left(I_{i}\right), \mu_{A_{j}}\left(I_{j}\right)\right) \\
\operatorname{NOT}\left(\mu_{A_{i}}\left(I_{i}\right)\right)=\mu_{\bar{A}_{i}}\left(I_{i}\right)=1-\mu_{A_{i}}\left(I_{i}\right) .
\end{gathered}
$$

Finally, the aggregated fuzzy output is mapped to its crisp form using widely accepted defuzzification method (i.e., center of gravity), defined as follows:

$$
\text { Center of gravity }(\mathrm{COG})=\frac{\sum_{j=1}^{p} z_{j} \cdot \mu_{C}\left(z_{j}\right)}{\sum_{j=1}^{p} \mu_{C}\left(z_{j}\right)},
$$

where $\mu_{C}\left(z_{j}\right)$ is the modified membership functions of fuzzy output set $C$ and $p$ is the number of points over which the modified fuzzy output is spread in $X$-axis. Note that Eqs. (2)(6) have been utilized in the proposed FLCSD scheme.

\subsection{System Description}

Without loss of generality, we envision a WRSN consisting of (i) $N$ number of sensor nodes, randomly distributed over a 2D monitoring region, (ii) $k(>1)$ number of homogeneous MCs, and (iii) an immobile base station (BS) or service station which not only collects sensory data but also responsible for maintenance and other services of the MCs. The BS is in charge of scheduling the MCs and also able to recharge the battery of an MC in negligible time. Each node is equipped with a rechargeable battery, having maximum energy capacity $E_{\max }$. We assume that the BS knows the location of each node and an event occurs at every node with the same possibility. Moreover, the nodes continuously sense the environment and send the sensed data to the BS in each data collection round. The network is also assumed to have no isolated node. Each MC is powered with energy capacity $E_{m c}$ that can be used to provide multinode energy transfer to the nearby nodes (i.e., nodes within the charging range) and also for its moving purpose. The working process of the system is as follows. Each node continuously keeps track of its remaining energy. When the energy level of a node falls below its charging threshold (which vary depending on its energy consumption rate (see Section 4.2)), it sends out a charging request to the assigned $\mathrm{MC}$. The charging request messages contain ID and location information of the node. Based on the network information, the $\mathrm{MC}$ determines the charging schedule of the nodes. A charging schedule $C_{\text {schedule }}$ is the visiting order of requesting nodes by which an MC recharges them. Mathematically, it can be shown as follows:

$$
C_{\text {schedule }}=\left\{B S \rightarrow s_{i} \rightarrow s_{i+1} \rightarrow \cdots \rightarrow s_{r}\right\},
$$

where node $s_{i+1}$ is scheduled to be recharged after $s_{i}$, and $r$ denotes the total requesting nodes in the request queue $\left(Q_{\text {req }}\right)$, i.e., $\left|Q_{\text {req }}\right|$. We use the same energy model as in [21], in which nodes may consume energy for transmitting, receiving, and computing tasks. However, energy consumption profiles of the nodes are not known in advance. Sensor nodes may have diverse energy consumption due to their uneven traffic loads,
TABLE 2

Important Notations

\begin{tabular}{ll}
\hline Notation & \\
\hline$s_{i}$ & Sensor node $i$ \\
$N(i)$ & Number of nodes in the $i$ th region \\
$N_{\text {coverage }}(i)$ & Number of nodes traversed by an MC in a recharging tour \\
$E_{\text {max }}$ & Battery capacity of the nodes \\
$E_{\text {mc }}$ & Battery capacity of the MCs \\
$D\left(s_{i}, m c\right)$ & Distance between $s_{i}$ and an MC \\
$D_{\text {max }}(B S)$ & Maximum possible distance of a node from the BS \\
$C N\left(s_{i}\right)$ & Critical node density of $s_{i}$ \\
$R E\left(s_{i}\right)$ & Residual energy of $s_{i}$ \\
$R E(m c)$ & Residual energy of an MC \\
$E C R\left(s_{i}\right)$ & Energy consumption rate of $s_{i}$ \\
$E_{\text {move }}$ & Moving energy consumption of the MCs per meter distance \\
$D_{\text {max }}$ & Maximum distance in the network between any two nodes \\
$C N_{\max }$ & Maximum critical node density of any node \\
$R E_{\text {max }}$ & Maximum residual energy of any node \\
$E C R_{\max }$ & Maximum energy consumption rate of any node \\
$Q_{\text {req }}$ & Queue in which charging requests are stored \\
$P_{r}\left(s_{i}\right)$ & Charging energy received per second by $s_{i}$ from an MC \\
$C_{\text {thres }}\left(s_{i}\right)$ & Charging threshold of $s_{i}$ \\
$T_{\text {coverage }}(i)$ & Time to recharge $N_{\text {coverage }}(i)$ nodes during a charging tour \\
$S P\left(s_{i}\right)$ & Scheduling probability of $s_{i}$ \\
$\eta$ & Charging energy efficiency rate \\
$\omega$ & Charging threshold of the MCs \\
\hline &
\end{tabular}

depending on the underlying application or routing protocol. 339 This means that the sensor nodes have heterogeneous and 340 dynamic rate of energy consumption.

Note that all the MCs are assumed to know this dynamic 342 rate of energy consumption of the nodes periodically using 343 the technique of energy notification messages as described 344 in [32]. Following the charging model in [21], the charging 345 energy received by $s_{i}$ (i.e., $\left.P_{r}\left(s_{i}\right)\right)$ is calculated as

$$
P_{r}\left(s_{i}\right)=\left\{\begin{array}{ll}
\eta \times c_{\text {rate }}, & \text { if } D\left(s_{i}, m c\right) \leq C_{r} \\
0, & \text { if } D\left(s_{i}, m c\right)>C_{r}
\end{array},\right.
$$

where $D\left(s_{i}, m c\right)$ is distance between $s_{i}$ and an MC, $c_{\text {rate }}$ is 349 the charging rate of the MCs, and $\eta(0<\eta<1)$ denotes 350 the charging energy efficiency rate. Since an MC usually has 351 a small charging range $C_{r}$ (e.g., $C_{r} \approx 2.7$ meters in [17]), we 352 assume that there is no difference in the recharging energy 353 among the nodes in terms of $\eta$ and $D\left(s_{i}, m c\right)$. In case of 354 multi-node charging, total stopping time of the $\mathrm{MC}$ (i.e., 355 $\left.t_{\text {charge }}\left(C N\left(s_{i}\right)\right)\right)$ for charging the nearby nodes, is calculated as 356

$$
t_{\text {charge }}\left(C N\left(s_{i}\right)\right)=\max _{s_{i} \in C N\left(s_{i}\right)}\left(t_{\text {charge }}\left(s_{i}\right)\right),
$$

where $t_{\text {charge }}\left(s_{i}\right)$ is the time taken by an $\mathrm{MC}$ to charge $s_{i}$, i.e., 359 $t_{\text {charge }}\left(s_{i}\right)=E_{\max } / P_{r}\left(s_{i}\right)$. We do not consider the handling 360 of routing the charging requests in the paper. For simplicity, 361 the routing time of charging requests is assumed to be negli- 362 gible in comparison to the traveling and charging time of 363 the MC [30]. Table 2 summarizes the important notations 364 used in the paper.

\section{Proposed Scheme}

The proposed scheme has the following basic operational 367 steps: (1) after network initialization, it first partitions the 368 network to evenly distribute the charging load of sensor 369 


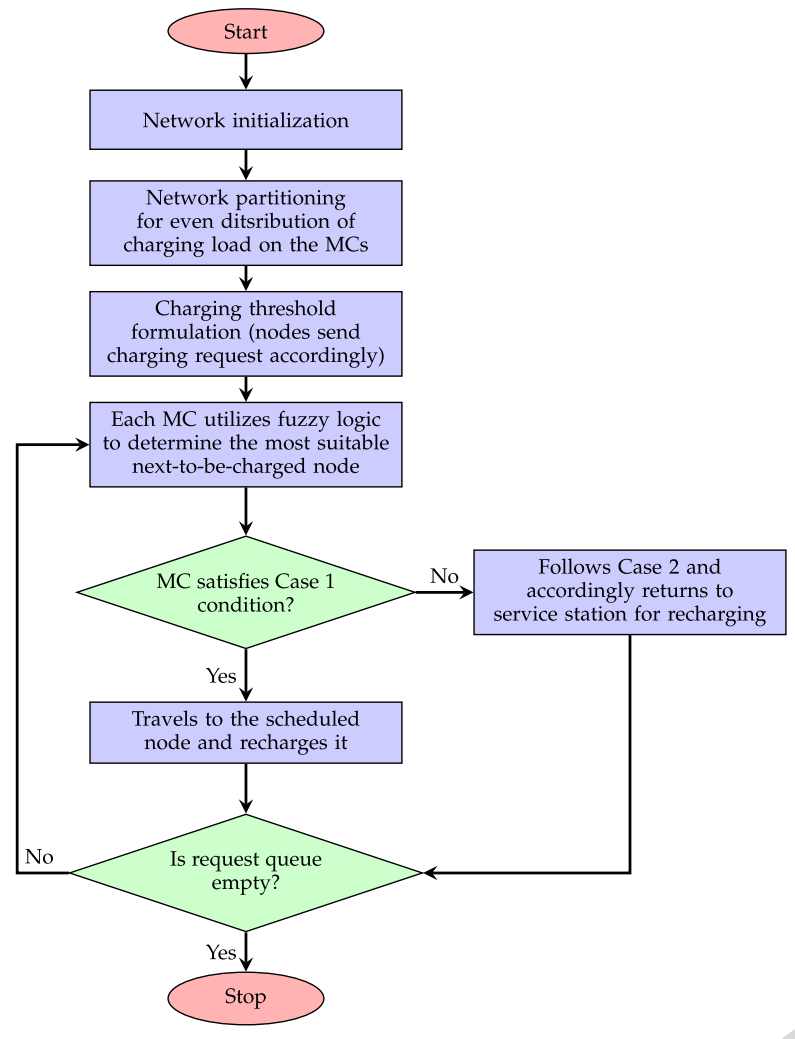

Fig. 2. Flowchart of the FLCSD scheme.

nodes among the MCs, (2) it then estimates a dynamic charging threshold for nodes based on their heterogeneous energy consumption rate, and (3) finally, it evaluates various network attributes jointly using fuzzy logic in order to determine most suitable next-to-be-charged node. All these aforementioned steps are presented in the form of a flow chart as shown in Fig. 2.

\subsection{Network Partitioning}

The goal of network partitioning is to assign the MCs to $k$ different regions such that each MC belongs to one region only. The main idea is to evenly distribute the traffic load of the nodes in network so as to balance the charging load among the MCs. This provides in-time charging response to the nodes with uneven energy consumption rates. Note that the proposed approach is not affected by the size as well as the density of the network. Besides, the BS performs network partitioning 385 periodically by following the proposed approach so as to effec- 386 tively deal with the network dynamics over the time. 387

The traffic loads of all the nodes are known to be a priori 388 by following any standard routing algorithm. By traffic load 389 of a node, we mean the total number of data packets for- 390 warded by that node to the BS in one data collection round. 391 The BS is assumed to be located at the center of the network. 392 Let TTL be the total traffic load of network and RTL be the 393 regional traffic load of a particular region. In general, there 394 are two following conditions for an efficient network 395 partitioning:

(i) If $\bmod (T T L, k)==0$, the network partitioning will 397 perform efficient traffic load balancing when the 398 $R T L$ of each region is $T T L / k$.

(ii) If $\bmod (T T L, k) \neq 0$, the network partitioning will 400 perform efficient traffic load balancing when each of 401 the $\bmod (T T L, k)$ regions has $R T L=(T T L / k)+1$ and 402 remaining regions have $R T L=T T L / k$.

Our network partitioning approach works as follows. For 404 the sake of first partitioned region, a base axis line is consid- 405 ered from the BS which intersects the rectangular network 406 horizontally (see Fig. 3b). Then, the angular distance of all 407 the nodes from the base axis line is computed. The angular 408 distance of the nodes is calculated by the formulas given in 409 Fig. 3c. Next, all the angular distances are sorted in the non- 410 decreasing order. Now, one by one all the nodes are selected 411 in the non-decreasing order of their angular distances until 412 $R T L \leq\left\lfloor\frac{T T L}{k}\right\rfloor$. All the nodes, satisfying this condition, belong 413 to the first region. Likewise, this process is repeated until all 414 nodes are covered and $k$ number of regions are formed. 415 Mathematically, it can be explained as follows. Let $A D\left(s_{i}\right) 416$ be the angular distance of $i$ th node from the base axis line 417 and $R^{\prime}(m)$ be the $m$ th region in the network. $T$ denotes the 418 set of nodes which have their angular distances in non- 419 decreasing order, i.e., $T=\left\{s_{1}, s_{2}, \ldots, s_{N}\right\}$, where $A D\left(s_{1}\right) \leq 420$ $A D\left(s_{2}\right) \leq \cdots \leq A D\left(s_{N}\right)$. Suppose $l$ number of nodes from set 421 $T$ are already distributed to $m$ number of regions $(m<k), 422$ then if there exist $j$ number of nodes $\left\{s_{l+1}, s_{l+2}, \ldots, s_{l+j}\right\}$ from 423 the remaining nodes, such that $R T L\left(=\sum_{c=l+1}^{l+j} T L_{c}\right) \leq\left\lfloor\frac{T T L}{k}\right\rfloor, 424$ then $\left\{s_{l+1}, s_{l+2}, \ldots, s_{l+j}\right\} \in R^{\prime}(m+1)$, where $T L_{c}$ is the traffic 425 load of $c$ th node. This means the nodes $\left\{s_{l+1}, s_{l+2}, \ldots, s_{l+j}\right\} 426$ belong to the region next to $R^{\prime}(m)$.

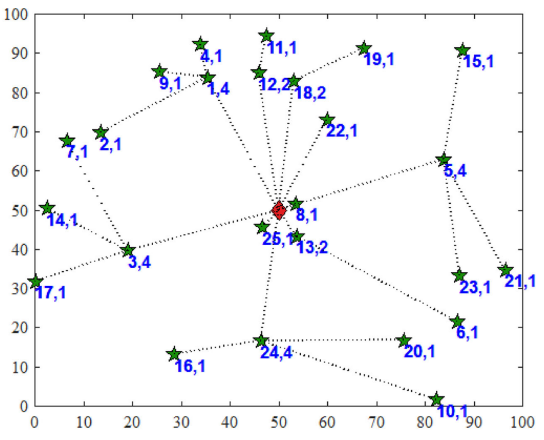

(a)

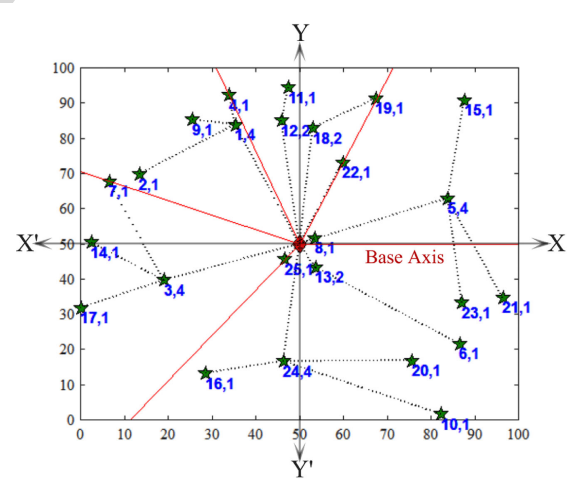

(b)

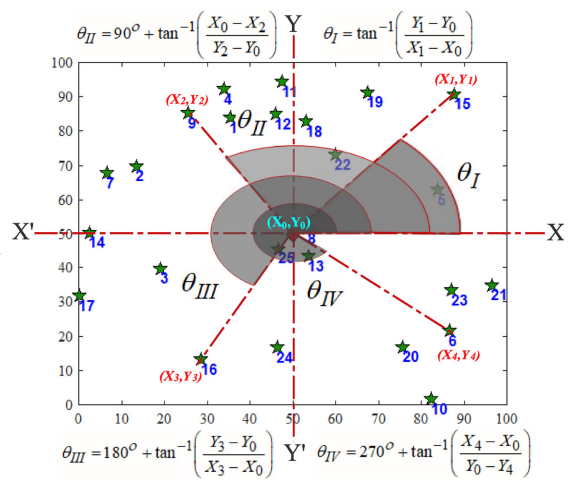

(c)

Fig. 3. Network partitioning approach with $k=5$ showing, (a) network scenario with 25 nodes, (b) five partitions with $R T L=8,6,6,7$, and 13 , and (c) calculation of the angular distances of nodes from base axis line. 


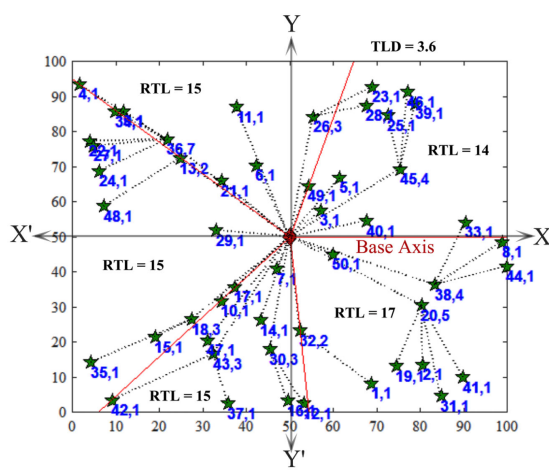

(a)

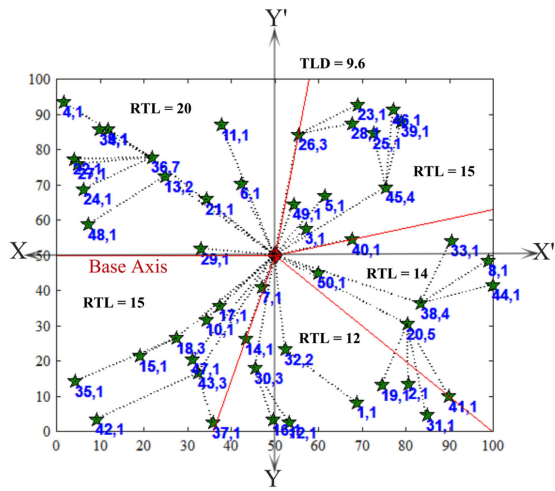

(d)

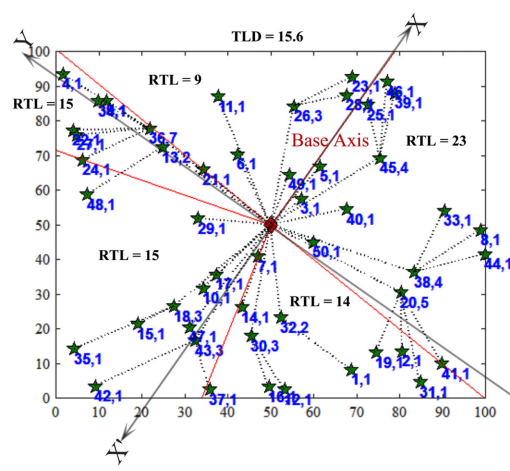

(b)

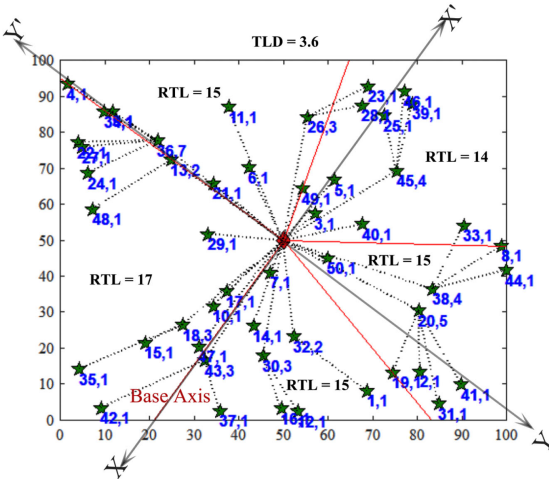

(e)

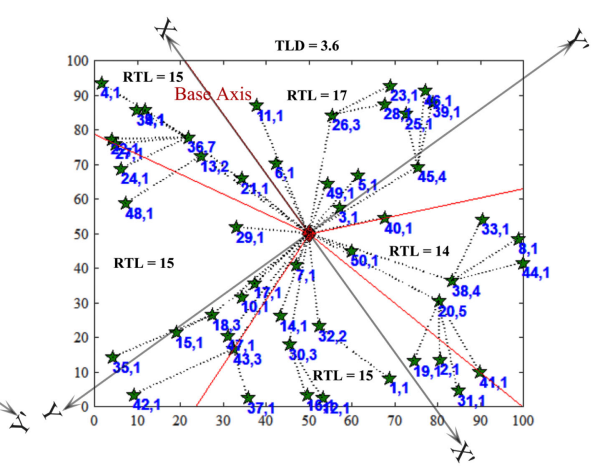

(c)

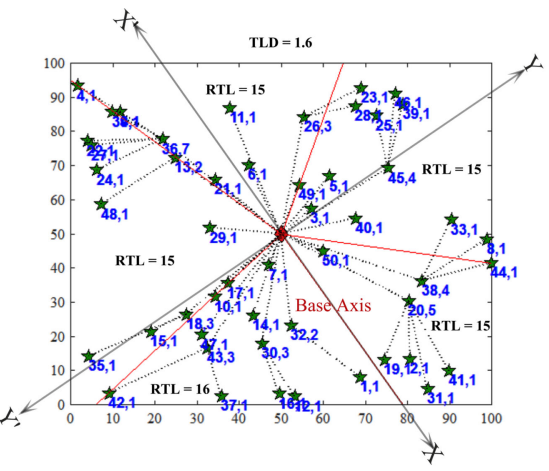

(f)

Fig. 4. Network partitioning approach with $k=5$ showing, (a) Base axis at $0^{\circ}$, (b) Base axis at $60^{\circ}$, (c) Base axis at $120^{\circ}$, (d) Base axis at $180^{\circ}$, (e) Base axis at $240^{\circ}$, and (f) Base axis at $300^{\circ}$.

For the sake of illustrating the network partitioning approach, we consider a random network scenario shown in Fig. 3a, in which 25 nodes are distributed randomly. In figure, nodes are represented along with their ID and traffic load. The TTL of the network is 40 . By assuming $k=5$, the $R T L$ should be $\left\lfloor\frac{40}{5}\right\rfloor=8$. Now, we pick nodes one by one in non-decreasing order of their angular distances until their $R T L$ becomes $8(R T L \leq 8)$. The nodes $5,8,15,19$, and 22 are selected for the first region as their $R T L=8$. This process is further repeated in a similar manner until all the five regions are formed. The final partitions are shown in Fig. 3b with $R T L=8,6,6,7$, and 13 , respectively. It is evident from the above example that a sparse WRSN may have a case when $R T L$ is not evenly balanced among the regions. Moreover, as the network density increases, considering different base axis lines may result in better traffic load distribution. In view of the above facts, our approach considers different base axis lines at different angular rotations while partitioning the network.

We consider another dense network scenario where base axis is rotated by $60^{\circ}$ to create $\left(\frac{360^{\circ}}{60^{\circ}}=\right) 6$ different partitioning instances which is shown in Figs. 4a, 4b, 4c, 4d, 4e, and 4f. Let $T L D$ be the total load deviation in the network and is defined as

$$
T L D=\sum_{i=1}^{k}\left|R T L_{a v g}-R T L_{i}\right|,
$$

where $R T L_{i}$ and $R T L_{\text {avg }}$ denote the $R T L$ of $i$ th region and the average $R T L$ of all the regions, respectively. Among all the partitioning instances (Figs. 4a, 4b, 4c, 4d, 4e, and 4f), we select the instance having minimum $T L D$ which is most 457 efficient partitioning as shown in Fig. $4 \mathrm{f}$ with $T L D=1.6 . \quad 458$

It can be further observed that in a highly dense WRSN, a 459 slight change in the base axis may lead to different partition- 460 ing results. Thus, our approach suggests to change the base 461 axis by $1^{\circ}$ for each partition and then checks for the best par- 462 titioning results with almost equal $R T L$ distribution. There 463 can be some cases in which more than one instances have 464 same minimum $T L D$, then the instance having minimum 465 $T D D$ is selected. Here, $T D D$ is the total density deviation in 466 the network and is defined as

$$
T D D=\sum_{i=1}^{k}\left|R N D_{\text {avg }}-R N D_{i}\right|,
$$

where $R N D_{i}$ denotes the regional node density, i.e., total 470 number of nodes in $i$ th region, and $R N D_{\text {avg }}$ denotes the 471 average node density of all the regions.

In case of more than one instances having minimum 473 $T D D$, any instance with minimum $T D D$ can be selected. 474 The $T L D$ of all the $\left(\frac{360^{\circ}}{1^{\circ}}=\right) 360$ instances for a random net- 475 work scenario is highlighted in Fig. 5a. In figure, the 476 highlighted red colored boxes show the range of rotated 477 base axis angles, where partitioning instances are created 478 with minimum $T L D(=1.6)$.

479

Here, we have shown only two instances (Figs. 5b and 5c) 480 having minimum $T L D$. The Fig. $5 \mathrm{c}$ is chosen to be final par- 481 titioning as it has minimum $T D D=4$. In Fig. 5 c, it can be 482 observed that traffic load is evenly distributed among all 483 the five partitioned regions. At the same time, each partition 484 of this instance has optimum node density distribution as 485 


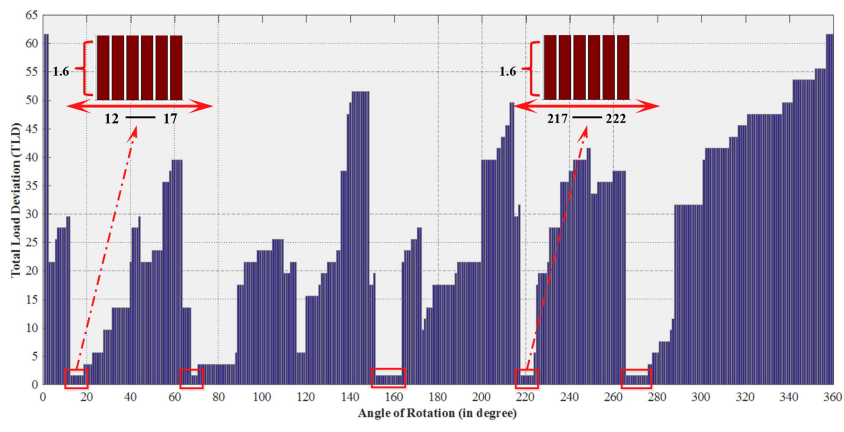

(a)

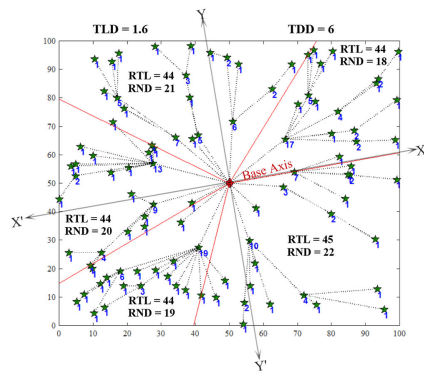

(b)

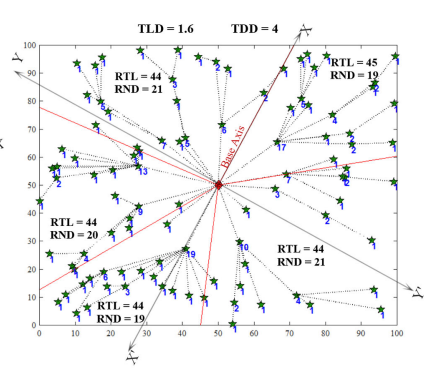

(c)
Fig. 5. Network partitioning approach with $k=5$ showing, (a) variation of $T L D$ with angle of rotation, (b) the partitioning instance with $T L D=1.6$ and $T D D=6$, (c) the partitioning instance with $T L D=1.6$ and $T D D=4$.

compared to other instances. After partitioning of the network, the BS assigns one $\mathrm{MC}$ in each region and notifies the nodes about ID of the MC assigned to them for sending the charging requests. Besides, each MC knows the location of nodes in its respective region.

\subsection{Threshold Formulation of Charging Request}

It is inarguable that unpredictable events occur in the network sporadically and thus energy consumption rates of nodes vary significantly. In such condition, fixed charging threshold for the nodes is not suitable for dynamic networks. A very low threshold results in response delay of charging requests, thereby causing more number of nodes to have premature death. On the other hand, too high threshold degrades the charging efficiency since too much time is spent on moving due to more frequent charging requests. Thus, formulating a reasonable charging threshold for the nodes is worth exploring so as to improve the charging performance in the network. Inspired by the above facts, an adaptive approach for calculating the charging threshold is proposed as follows.

Let us consider $i$ th region in the network, where $N(i)$ be the total number of nodes, and $N_{\text {coverage }}(i)$ be the number of nodes which can be traversed by an MC in a recharging tour. Let $E_{\text {coverage }}$ be the total energy consumed by the $\mathrm{MC}$ in order to visit $N_{\text {coverage }}(i)$ nodes which is defined as

$$
E_{\text {coverage }}=\left(N_{\text {coverage }}(i)+1\right) \times D_{\text {average }} \times E_{\text {move }},
$$

where

$$
D_{\text {average }}=\frac{1}{N(i) \times(N(i)+1)} \sum_{x=1}^{N(i)+1} \sum_{y=1, y \neq x}^{N(i)+1} D\left(s_{x}, s_{y}\right)
$$

Here, $D_{\text {average }}$ is the average euclidean distance between any 516 two nodes, $\left(N_{\text {coverage }}(i)+1\right)$ represents $N_{\text {coverage }}(i)$ nodes 517 including the BS, $D\left(s_{x}, s_{y}\right)$ denotes the euclidean distance 518 between node $s_{x}$ and $s_{y}$, and $E_{\text {move }}$ is the energy consump- 519 tion of MC per meter traveling distance. Next, $N_{\text {coverage }}(i) 520$ can be computed as follows:

$$
N_{\text {coverage }}(i)=\left\lfloor\frac{E_{m c}-E_{\text {coverage }}}{E_{\text {max }}}\right\rfloor,
$$

where $E_{m c}$ and $E_{\max }$ are the battery capacity of the MCs and 524 the nodes, respectively. In Eq. (14), the value of $E_{\text {coverage }}$ is 525 substituted from Eq. (12) to calculate $N_{\text {coverage }}(i)$. Then, the 526 total time $\left(T_{\text {coverage }}(i)\right)$ taken by the $\mathrm{MC}$ to recharge 527 $N_{\text {coverage }}(i)$ nodes during its tour is computed as follows:

$$
\begin{aligned}
T_{\text {coverage }}(i)= & \left(N_{\text {coverage }}(i)+1\right) \times\left(\frac{D_{\text {average }}}{v_{\text {mc }}}\right) \\
& +N_{\text {coverage }}(i) \times\left(\frac{E_{\text {max }}}{\eta \times c_{\text {rate }}}\right) .
\end{aligned}
$$

For the sake of satisfying all the energy demands, the 531 MC needs to complete $\left[\frac{N(i)}{N_{\text {coverage }}(i)}\right]$ charging tours. Thus, the 532 maximum waiting time of a node can be computed as 533 $T_{\text {coverage }}(i) \times\left[\frac{N(i)}{N_{\text {coverage }}(i)}\right\rceil$. In view of the above facts, the charg- 534 ing threshold of $s_{i}$ (i.e., $C_{\text {thres }}\left(s_{i}\right)$ ) is calculated as follows: 535

$$
C_{\text {thres }}\left(s_{i}\right)=\operatorname{ECR}\left(s_{i}\right) \times T_{\text {coverage }}(i) \times\left\lceil\frac{N(i)}{N_{\text {coverage }}(i)}\right\rceil \text {. (16) }
$$

\subsection{Charging Schedule Determination}

As discussed earlier, each MC computes the charging sched- 540 ule of its regional nodes. When an $\mathrm{MC}$ receives the charging 541 requests of its regional nodes, it stores them into $Q_{\text {req }}$. Based 542 on the network information, the MC stores the values of all 543 the network attributes. Note that the critical node density for 544 a node can be computed as the total number of requesting 545 nodes which fall within the charging range of that node. 546 Thereupon, scheduling probability of each requesting node 547 is computed by applying the FIS in order to determine the 548 most suitable next-to-be-charged node. The MC visits that 549 node and recharges it along with the other requesting nodes 550 within the charging range. After completion of charging, the 551 MC deletes the recharged nodes from the $Q_{\text {req }}$. Then, the MC 552 again updates the network information of remaining nodes 553 and computes the next most suitable next-to-be-charged 554 node. Likewise, all the requesting nodes are scheduled and 555 recharged one by one iteratively. Thus, we can say that 556 charging schedule determination follows a real-time and 557 dynamic process. If in-between, new charging requests are 558 triggered, then they wait until previous charging schedule is 559 completed. Before serving a scheduled node $s_{i}$, an MC con- 560 siders the following two cases.

Case 1: Let $D_{\max }(B S)$ be the maximum possible distance 562 of a node from the BS. If $\mathrm{MC}^{\prime} \mathrm{s}$ residual energy is higher 563 than threshold value $\omega$ (where $\left.\omega \geq E_{\text {move }} \times D_{\text {max }}(B S)\right)$, then 564 it continues to recharge the scheduled nodes.

Case 2: If $\mathrm{MC}$ has remaining energy less than the $\omega, 566$ nodes in $Q_{r e q}$ are judged whether they meet the following 567 constraint 


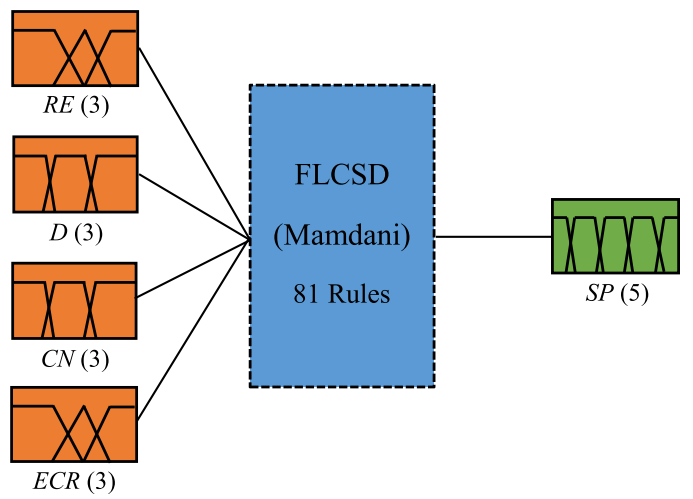

Fig. 6. The FIS of FLCSD scheme: 4 inputs, 1 output, 81 rules.

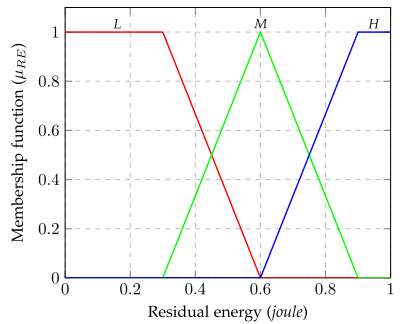

(a)

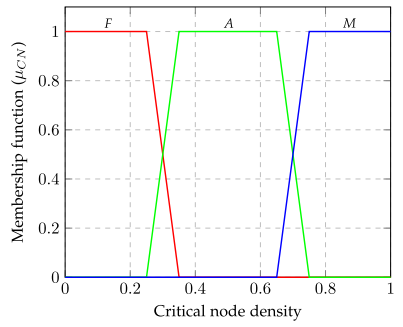

(c)

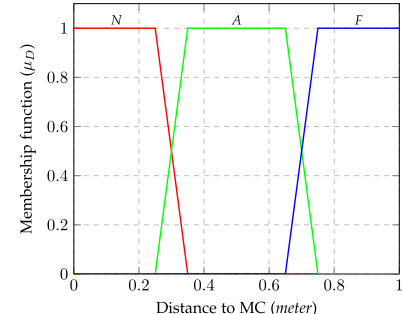

(b)

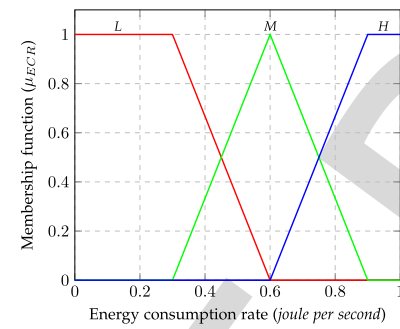

(d)
Fig. 7. Fuzzy input membership functions: (a) Residual energy, (b) Distance to MC, (c) Critical node density, and (d) Energy consumption rate.

$$
\begin{aligned}
& E_{\text {move }} \times D\left(s_{i}, B S\right) \leq R E(m c)(t)-\left(E_{\text {move }} \times D\left(s_{i}, m c\right)\right) \\
& -\left(E_{\text {max }}-R E\left(s_{i}\right)\left(t+t^{\prime}\right)\right),
\end{aligned}
$$

where $R E(m c)(t)$ denotes the residual energy of $\mathrm{MC}$ at time $t, t^{\prime}$ is the time when MC reaches the node $s_{i}, R E\left(s_{i}\right)\left(t+t^{\prime}\right)$ is the remaining energy of $s_{i}$ at time $\left(t+t^{\prime}\right)$.

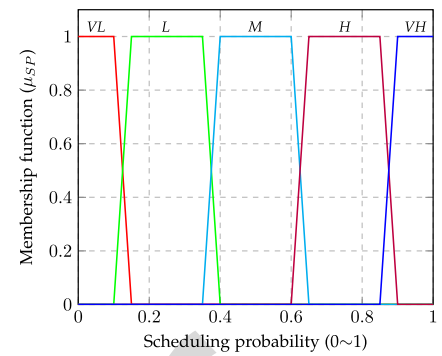

Fig. 8. Fuzzy output membership function: Scheduling probability.

In summary, the MC first examines if after recharging the 574 next scheduled node, it has sufficient remaining energy for 575 reaching the BS. Nodes which satisfy the constraint (Eq. (17)) 576 are chosen as the candidate nodes from which the next node 577 is scheduled for recharging by following the proposed 578 approach. If no requesting node satisfies the above con- 579 straint, then the MC do not entertain any other charging 580 request and instantly returns to the BS for its recharging.

Next, we elucidate the process of determining the next-to- 582 be-charged node using the FLCSD scheme. The FIS in our 583 scheme consists of 4-inputs and 1-output fuzzy linguistic vari- 584 ables, namely residual energy $(R E)$, distance to $M C(D)$, critical 585 node density $(C N)$, energy consumption rate $(E C R)$, and schedul- 586 ing probability $(S P)$, respectively. The design of MAMDANI 587 FIS is presented in Fig. 6 and its pseudo-code is delineated in 588 Procedure FUZZY. Figs. 7 and 8 depict trapezoidal and trian- 589 gular membership functions and show that these fuzzy varia- 590 bles are represented by more than one fuzzy linguistic values. 591 The input and output fuzzy variables and their corresponding 592 linguistic values are presented in Tables 3 and 4, respectively. 593

The proposed FLCSD scheme uses following trapezoidal 594 and triangular membership functions

$$
\begin{aligned}
& \text { Trapezoidal }(x ; a, b, c, d)=\left\{\begin{array}{cl}
0 & , x \leq a \\
\frac{x-a}{b-a} & , a \leq x \leq b \\
1 & , b \leq x \leq c \\
\frac{d-x}{d-c} & , c \leq x \leq d \\
0 & , d \leq x
\end{array}\right. \\
& \text { Triangular }(x ; a, b, c)=\left\{\begin{array}{cl}
0 & , x \leq a \\
\frac{x-a}{b-a} & , a \leq x \leq b \\
\frac{c-x}{c-b} & , b \leq x \leq c \\
0 & , c \leq x
\end{array}\right.
\end{aligned}
$$

\begin{tabular}{|c|c|c|}
\hline Input variable & Linguistic values & Membership function range \\
\hline Residual energy $(R E)$ & $\begin{array}{l}\operatorname{low}(L) \\
\text { medium }(M) \\
\operatorname{high}(H)\end{array}$ & $\begin{array}{c}{\left[0,0,0.3^{*} E_{\max }, 0.6^{*} E_{\max }\right]} \\
{\left[0.3^{*} E_{\max }, 0.6^{*} E_{\max }, 0.9^{*} E_{\max }\right]} \\
{\left[0.6^{*} E_{\max }, 0.9^{*} E_{\max }, E_{\max }, E_{\max }\right]}\end{array}$ \\
\hline Distance to $M C(D)$ & $\begin{array}{l}\operatorname{near}(N) \\
\operatorname{average}(A) \\
\operatorname{far}(F)\end{array}$ & $\begin{array}{c}{\left[0,0,0.25^{*} D_{\max }, 0.35^{*} D_{\max }\right]} \\
{\left[0.25^{*} D_{\max }, 0.35^{*} D_{\max }, 0.65^{*} D_{\max }, 0.75^{*} D_{\max }\right]} \\
{\left[0.65^{*} D_{\max }, 0.75^{*} D_{\max }, D_{\max }, D_{\max }\right]}\end{array}$ \\
\hline Critical node density (CN) & $\begin{array}{c}\text { few }(F) \\
\text { average }(A) \\
\text { many }(M)\end{array}$ & $\begin{array}{c}{\left[0,0,0.25^{*} C N_{\max }, 0.35^{*} C N_{\max }\right]} \\
{\left[0.25^{*} C N_{\max }, 0.35^{*} C N_{\max }, 0.65^{*} C N_{\max }, 0.75^{*} C N_{\max }\right]} \\
{\left[0.65^{*} C N_{\max }, 0.75^{*} C N_{\max }, C N_{\max }, C N_{\max }\right]}\end{array}$ \\
\hline Energy consumption rate (ECR) & $\begin{array}{l}\operatorname{low}(L) \\
\text { medium }(M) \\
\operatorname{high}(H)\end{array}$ & $\begin{array}{c}{\left[0,0,0.3^{*} E C R_{\max }, 0.6^{*} E C R_{\max }\right]} \\
{\left[0.3^{*} E C R_{\max }, 0.6^{*} E C R_{\max }, 0.9^{*} E C R_{\max }\right]} \\
{\left[0.6^{*} E C R_{\max }, 0.9^{*} E C R_{\max }, E C R_{\max }, E C R_{\max }\right]}\end{array}$ \\
\hline
\end{tabular}

TABLE 3

Input Variables With Their Linguistic Values and Corresponding Membership Function Ranges 
TABLE 4

Output Variable With its Linguistic Values and Membership Function Ranges

\begin{tabular}{lcc}
\hline Output variable & Linguistic values & Membership function range \\
\hline Scheduling probability $(S P)$ & very low $(V L)$ & {$[0,0,0.1,0.15]$} \\
& low $(L)$ & {$[0.1,0.15,0.35,0.4]$} \\
& moderate $(M)$ & {$[0.35,0.4,0.6,0.65]$} \\
& $\operatorname{high}(H)$ & {$[0.6,0.65,0.85,0.9]$} \\
& very high $(V H)$ & {$[0.85,0.9,1,1]$} \\
\hline
\end{tabular}

where $x$ is the crisp input, and $a, b, c, d$ are membership function ranges of the fuzzy variables. Note that these values are independent of network size and node count in the proposed scheme.

The complete rule set in the proposed scheme, consists of $3^{4}=81$ rules as presented in Table 5 . We now transform the crisp data inputs to the fuzzy inputs using the Trapezoidal and Triangular membership functions as given in Eqs. (18) and (19), respectively. Let us consider a random charging request for a node in the $Q_{\text {req, }}$ e.g., $R E=1.52 \mathrm{KJ}, D=$ $32 \mathrm{~m}, C N=1$, and $E C R=0.02 \mathrm{~J} / \mathrm{s}$. Here, we consider $E_{\max }=10.8 K J, D_{\max }=125.09 \mathrm{~m}, C N_{\max }=5$, and $E C R_{\max }=$ $0.08 \mathrm{~J} / \mathrm{s}$. Then, the fuzzy inputs of different linguistic variables are calculated from the aforesaid crisp inputs using Eqs. (18) and (19) as presented in Table 6.

Now, the fuzzy outputs for each fuzzy rule are evaluated from fuzzy inputs (using Eqs. (2)-(5)). The evaluated values
TABLE 6

Calculation of Fuzzy Inputs From Crisp Inputs Using Input Fuzzy Membership Functions

\begin{tabular}{lccc}
\hline $\operatorname{low}(R E)=1$ & $\operatorname{near}(D)=0.942$ & $\operatorname{few}(C N)=1$ & $\operatorname{low}(E C R)=1$ \\
$\operatorname{medium}(R E)=0$ & $\operatorname{average}(D)=0.058$ & $\operatorname{average}(C N)=0$ & $\operatorname{medium}(E C R)=0$ \\
$\operatorname{high}(R E)=0$ & $\operatorname{far}(D)=0$ & $\operatorname{many}(C N)=0$ & $\operatorname{high}(E C R)=0$ \\
\hline
\end{tabular}

for all the fuzzy rules are presented in Table 7. For example, 618 the fuzzy output for rule $R_{1}$ is calculated as follows:

$$
\begin{aligned}
\Rightarrow \operatorname{eval}\left(R_{1}\right) & =\operatorname{MIN}(\operatorname{low}(R E), \text { near }(D), \text { few }(C N), \operatorname{low}(E C R)) \\
& =\text { moderate }(S P)=0.942 .
\end{aligned}
$$

The aggregated fuzzy output is generated from the modi- 623 fied fuzzy outputs in Table 7. The fuzzy membership ranges of 624 $S P$ are span over the intervals, such as VL=NULL, $L=625$ [0.1, 0.1029, 0.3971, 0.4], $M=[0.35,0.3971,0.6029,0.65], H=626$ NULL, and $V H=N U L L$. Finally, the crisp output (i.e., defuzzi- 627 fication point) is calculated as 0.509 (see Fig. 9) from the aggre- 628 gated fuzzy output by using center of gravity defuzzification 629 method (Eq. (6)). The computed crisp output for a node is the 630 value of its scheduling probability, i.e., $S P$ value. It is to note 631 here that the node having the highest $S P$ value is scheduled as 632 the next-to-be-charged node. In case of more than one node 633 having same $S P$ value, the node closest to the MC is given 634 higher priority for recharging the next. The pseudo-code of the 635 proposed scheme is shown in Algorithm 1.

TABLE 5

\begin{tabular}{|c|c|c|c|c|c|c|c|c|c|c|c|c|c|c|c|c|c|}
\hline \multirow{2}{*}{$R_{\#}$} & \multicolumn{4}{|c|}{ Inputs } & \multirow{2}{*}{$\begin{array}{l}\text { Output } \\
S P\end{array}$} & \multirow{2}{*}{$R_{\#}$} & \multicolumn{4}{|c|}{ Inputs } & \multirow{2}{*}{$\begin{array}{c}\text { Output } \\
S P\end{array}$} & \multirow{2}{*}{$R_{\#}$} & \multicolumn{4}{|c|}{ Inputs } & \multirow{2}{*}{$\begin{array}{c}\text { Output } \\
S P\end{array}$} \\
\hline & $\overline{R E}$ & $D$ & $\mathrm{CN}$ & $\overline{E C R}$ & & & $\overline{R E}$ & $D$ & $\mathrm{CN}$ & $\overline{E C R}$ & & & $\overline{R E}$ & $D$ & $\mathrm{CN}$ & $\overline{E C R}$ & \\
\hline$\overline{R_{1}}$ & $L$ & $N$ & $\bar{F}$ & $L$ & $M$ & $R_{28}$ & $M$ & $N$ & $F$ & $L$ & $L$ & $R_{55}$ & $H$ & $N$ & $\bar{F}$ & $L$ & $\overline{V L}$ \\
\hline$R_{2}$ & $L$ & $N$ & $F$ & $M$ & $M$ & $R_{29}$ & $M$ & $N$ & $F$ & $M$ & $M$ & $R_{56}$ & $H$ & $N$ & $\mathrm{~F}$ & $M$ & VL \\
\hline$R_{3}$ & $L$ & $N$ & $F$ & $H$ & $V H$ & $R_{30}$ & $M$ & $N$ & $F$ & $H$ & $H$ & $R_{57}$ & $H$ & $N$ & $F$ & $H$ & $L$ \\
\hline$R_{4}$ & $L$ & $N$ & A & $L$ & $M$ & $R_{31}$ & $M$ & $N$ & $A$ & $L$ & $M$ & $R_{58}$ & $H$ & $N$ & $A$ & $L$ & $V L$ \\
\hline$R_{5}$ & $L$ & $N$ & $A$ & $M$ & $H$ & $R_{32}$ & $M$ & $N$ & $A$ & $M$ & $H$ & $R_{59}$ & $H$ & $N$ & $A$ & $M$ & $L$ \\
\hline$R_{6}$ & $L$ & $N$ & $A$ & $H$ & $V H$ & $R_{33}$ & $M$ & $N$ & $A$ & $H$ & $H$ & $R_{60}$ & $H$ & $N$ & $A$ & $H$ & $M$ \\
\hline$R_{7}$ & $L$ & $N$ & $M$ & $L$ & $H$ & $R_{34}$ & $M$ & $N$ & $M$ & $L$ & $M$ & $R_{61}$ & $H$ & $N$ & $M$ & $L$ & $L$ \\
\hline$R_{8}$ & $L$ & $N$ & $M$ & $M$ & VH & $R_{35}$ & $M$ & $N$ & $M$ & $M$ & $H$ & $R_{62}$ & $H$ & $N$ & $M$ & $M$ & $M$ \\
\hline$R_{9}$ & $L$ & $N$ & $M$ & $H$ & $V H$ & $R_{36}$ & M & $N$ & $M$ & $H$ & $V H$ & $R_{63}$ & $H$ & $N$ & $M$ & $H$ & $H$ \\
\hline$R_{10}$ & $L$ & A & $F$ & $L$ & $L$ & $R_{37}$ & $M$ & $A$ & $F$ & $L$ & $L$ & $R_{64}$ & $H$ & A & $F$ & $L$ & $V L$ \\
\hline$R_{11}$ & $L$ & A & $F$ & $M$ & $L$ & $R_{38}$ & $M$ & $A$ & $F$ & $M$ & $L$ & $R_{65}$ & $H$ & A & $F$ & $M$ & $V L$ \\
\hline$R_{12}$ & $L$ & A & $F$ & $H$ & $M$ & $R_{39}$ & $M$ & $A$ & $F$ & $H$ & $H$ & $R_{66}$ & $H$ & A & $F$ & $H$ & $L$ \\
\hline$R_{13}$ & $L$ & $A$ & A & $L$ & $L$ & $R_{40}$ & $M$ & $A$ & $A$ & $L$ & $M$ & $R_{67}$ & $H$ & $A$ & $A$ & $L$ & $V L$ \\
\hline$R_{14}$ & $L$ & A & A & $M$ & $M$ & $R_{41}$ & $M$ & $A$ & $A$ & $M$ & $M$ & $R_{68}$ & $H$ & A & $A$ & $M$ & $L$ \\
\hline$R_{15}$ & $L$ & A & $A$ & $H$ & $H$ & $R_{42}$ & $M$ & $A$ & $A$ & $H$ & $H$ & $R_{69}$ & $H$ & A & $A$ & $H$ & $M$ \\
\hline$R_{16}$ & $L$ & A & $M$ & $L$ & M & $R_{43}$ & $M$ & $A$ & $M$ & $L$ & $M$ & $R_{70}$ & $H$ & A & $M$ & $L$ & $L$ \\
\hline$R_{17}$ & $L$ & A & $M$ & $M$ & $H$ & $R_{44}$ & $M$ & $A$ & $M$ & $M$ & $M$ & $R_{71}$ & $H$ & A & $M$ & $M$ & $L$ \\
\hline$R_{18}$ & $L$ & $A$ & $M$ & $H$ & $V H$ & $R_{45}$ & $M$ & $A$ & $M$ & $H$ & $H$ & $R_{72}$ & $H$ & $A$ & $M$ & $H$ & $M$ \\
\hline$R_{19}$ & $L$ & $F$ & $F$ & $L$ & $L$ & $R_{46}$ & $M$ & $F$ & $F$ & $L$ & $L$ & $R_{73}$ & $H$ & $F$ & $F$ & $L$ & $V L$ \\
\hline$R_{20}$ & $L$ & $F$ & $F$ & $M$ & $L$ & $R_{47}$ & $M$ & $F$ & $F$ & $M$ & $L$ & $R_{74}$ & $H$ & $F$ & $F$ & $M$ & $V L$ \\
\hline$R_{21}$ & $L$ & $F$ & $F$ & $H$ & $M$ & $R_{48}$ & $M$ & $F$ & $F$ & $H$ & $M$ & $R_{75}$ & $H$ & $F$ & $F$ & $H$ & $L$ \\
\hline$R_{22}$ & $L$ & $F$ & $A$ & $L$ & $L$ & $R_{49}$ & $M$ & $F$ & $A$ & $L$ & $M$ & $R_{76}$ & $H$ & $F$ & $A$ & $L$ & $V L$ \\
\hline$R_{23}$ & $L$ & $F$ & $A$ & $M$ & $L$ & $R_{50}$ & $M$ & $F$ & $A$ & $M$ & $M$ & $R_{77}$ & $H$ & $F$ & $A$ & $M$ & $L$ \\
\hline$R_{24}$ & $L$ & $F$ & $A$ & $H$ & $H$ & $R_{51}$ & $M$ & $F$ & $A$ & $H$ & $H$ & $R_{78}$ & $H$ & $F$ & $A$ & $H$ & $L$ \\
\hline$R_{25}$ & $L$ & $F$ & $M$ & $L$ & $L$ & $R_{52}$ & $M$ & $F$ & $M$ & $L$ & $M$ & $R_{79}$ & $H$ & $F$ & $M$ & $L$ & $L$ \\
\hline$R_{26}$ & $L$ & $F$ & $M$ & $M$ & $M$ & $R_{53}$ & $M$ & $F$ & $M$ & $M$ & $M$ & $R_{80}$ & $H$ & $F$ & $M$ & $M$ & $L$ \\
\hline$R_{27}$ & $L$ & $F$ & $M$ & $H$ & $H$ & $R_{54}$ & $M$ & $F$ & $M$ & $H$ & $H$ & $R_{81}$ & $H$ & $F$ & $M$ & $H$ & $M$ \\
\hline
\end{tabular}

Fuzzy IF-THEN Rules for Scheduling Probability Determination 
TABLE 7

Fuzzy Rules Evaluation

\begin{tabular}{cccccccccccc}
\hline$R_{\#}$ & eval $\left(R_{\#}\right)$ & $R_{\#}$ & eval $\left(R_{\#}\right)$ & $R_{\#}$ & eval $\left(R_{\#}\right)$ & $R_{\#}$ & eval $\left(R_{\#}\right)$ & $R_{\#}$ & eval $\left(R_{\#}\right)$ & $R_{\#}$ & eval $\left(R_{\#}\right)$ \\
\hline$R_{1}$ & 0.942 & $R_{15}$ & 0 & $R_{29}$ & 0 & $R_{43}$ & 0 & $R_{57}$ & 0 & $R_{71}$ & 0 \\
$R_{2}$ & 0 & $R_{16}$ & 0 & $R_{30}$ & 0 & $R_{44}$ & 0 & $R_{58}$ & 0 & $R_{72}$ & 0 \\
$R_{3}$ & 0 & $R_{17}$ & 0 & $R_{31}$ & 0 & $R_{45}$ & 0 & $R_{59}$ & 0 & $R_{73}$ & 0 \\
$R_{4}$ & 0 & $R_{18}$ & 0 & $R_{32}$ & 0 & $R_{46}$ & 0 & $R_{60}$ & 0 & $R_{74}$ & 0 \\
$R_{5}$ & 0 & $R_{19}$ & 0 & $R_{33}$ & 0 & $R_{47}$ & 0 & $R_{61}$ & 0 & $R_{75}$ & 0 \\
$R_{6}$ & 0 & $R_{20}$ & 0 & $R_{34}$ & 0 & $R_{48}$ & 0 & $R_{62}$ & 0 & $R_{76}$ & 0 \\
$R_{7}$ & 0 & $R_{21}$ & 0 & $R_{35}$ & 0 & $R_{49}$ & 0 & $R_{63}$ & 0 & $R_{77}$ & 0 \\
$R_{8}$ & 0 & $R_{22}$ & 0 & $R_{36}$ & 0 & $R_{50}$ & 0 & $R_{64}$ & 0 & $R_{78}$ & 0 \\
$R_{9}$ & 0 & $R_{23}$ & 0 & $R_{37}$ & 0 & $R_{51}$ & 0 & $R_{65}$ & 0 & $R_{79}$ & 0 \\
$R_{10}$ & 0.058 & $R_{24}$ & 0 & $R_{38}$ & 0 & $R_{52}$ & 0 & $R_{66}$ & 0 & $R_{80}$ & 0 \\
$R_{11}$ & 0 & $R_{25}$ & 0 & $R_{39}$ & 0 & $R_{53}$ & 0 & $R_{67}$ & 0 & $R_{81}$ & 0 \\
$R_{12}$ & 0 & $R_{26}$ & 0 & $R_{40}$ & 0 & $R_{54}$ & 0 & $R_{68}$ & 0 & - & - \\
$R_{13}$ & 0 & $R_{27}$ & 0 & $R_{41}$ & 0 & $R_{55}$ & 0 & $R_{69}$ & 0 & - & - \\
$R_{14}$ & 0 & $R_{28}$ & 0 & $R_{42}$ & 0 & $R_{56}$ & 0 & $R_{70}$ & 0 & - & - \\
\hline
\end{tabular}

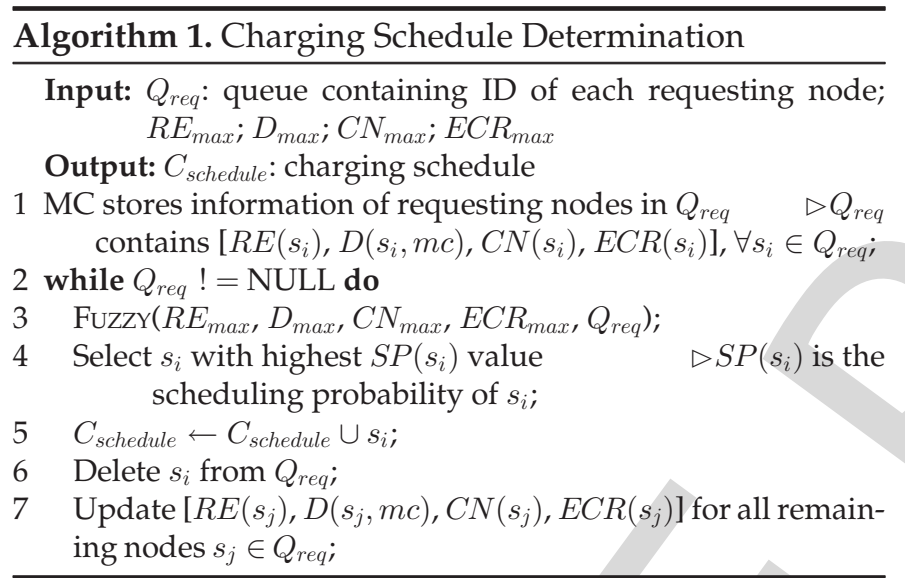

\section{Procedure FUZZY $\left(R E_{\max }, D_{\max }, C N_{\max }, E C R_{\max }, Q_{\text {req }}\right)$}

Input: $Q_{\text {req }}$ (stored crisp inputs); $r:\left|Q_{\text {req }}\right| ; q$ : number of fuzzy linguistic variables; $m$ : number of fuzzy linguistic values

Output: $S P$ : set of scheduling probabilities (crisp output)

1 Define input and output fuzzy linguistic variables;

2 Define fuzzy linguistic values for each linguistic variables;

3 for $i \leftarrow 1$ to $m^{q}$ do

4 Establish fuzzy rule $R_{i}$

$5 S P \longleftarrow \phi$

6 for $i \leftarrow 1$ to $r$ do

$7 \quad$ for $j \leftarrow 1$ to $q$ do

$8 \quad$ for $k \leftarrow 1$ for $m$ do

9 Mapping crisp input $\left[R E\left(s_{i}\right), D\left(s_{i}, m c\right), C N\left(s_{i}\right)\right.$, $\left.E C R\left(s_{i}\right)\right]$ to fuzzy input using input membership functions;

10 Aggregate $\longleftarrow \phi$

11 for $i \leftarrow 1$ to $m^{q}$ do

12 Evaluate fuzzy rule $R_{i}$, i.e., eval $\left(R_{i}\right) \triangleright$ using Eqs. (2) - (5); 13 Aggregate $\longleftarrow$ Aggregate $\cup \operatorname{eval}\left(R_{i}\right) \quad \triangleright$ Aggregate each evaluated fuzzy outputs;

$14 \quad$ Calculate crisp output $S P\left(s_{i}\right) \quad \triangleright$ using Eq. (6);

$15 S P \longleftarrow S P \cup S P\left(s_{i}\right)$

16 return $S P$

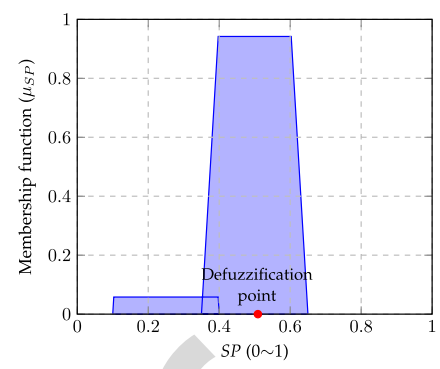

Fig. 9. Aggregated fuzzy output.

\section{Simulation Analysis}

675

To testify the benefits of the proposed scheme, extensive 676 simulations have been carried out. The charging schemes 677 proposed in [20] and [21] have been used for comparing 678 and evaluating the proposed FLCSD scheme. Both the 679 schemes [20] and [21] follow an on-demand multi-node 680 charging architecture, employing multiple MCs. We have 681 used the scheme [20] as UCMC and the scheme [21] as 682 OMC_TSP in the interest of depicting the performance 683 results. For fair comparisons, it is assumed that there is no 684 aggregated electromagnetic radiation (EMR) between 685 nearby MCs. We mainly focus on three performance met- 686 rics, namely energy usage efficiency, survival rate, and average 687 charging latency in order to judge the efficacy of the pro- 688 posed scheme.

\subsection{Simulation Environment}

690

We have considered a dense WRSN with 400 to 1000 nodes 691 randomly placed in $100 \times 100 \mathrm{~m}^{2}$ region. The BS is located 692 at the center of the network. We have assumed the follow- 693 ing parametric values for the simulation. Each node has 694 power capacity of $10.8 \mathrm{KJ}$ [27]. The maximum energy of each 695 $\mathrm{MC}$ is $4000 \mathrm{KJ}$ [27]. Each MC charges at the rate of $5 \mathrm{~J} / \mathrm{s}$ [21] 696 with $\eta \approx 0.68$ [17]. Each MC has moving speed of $v_{m c}$ with 697 per meter energy consumption of $600 J$ [24], [27]. The nodes 698 randomly consume between 0.01 to $0.05 \mathrm{~J} / \mathrm{s}$ for sensing, com- 699 munication, and computation tasks in order to emulate their 700 non-predictable energy consumption [21]. The simulations 701 are performed in MATLAB version R2016a using a system of 702 4 GB RAM and Intel Core 2 Duo processor. We have consid- 703 ered the average of 20 random simulation instances for 704 depicting the result in each figure for the sake of fair compar- 705 isons. The important simulation parameters with their val- 706 ues are listed in Table 8.

\subsection{Performance Evaluation}

708

In this section, we assess the impact of various network 709 parameters (i.e., number of nodes, simulation time, and moving 710 speed of $M C$ ) on the performance metrics.

\subsubsection{Energy Usage Efficiency}

Energy usage efficiency (EUE) is defined as the ratio between 713 the energy obtained by nodes and the overall energy trans- 714 ferred from the BS to the MCs [33]. The EUE plays a vital role 715 in achieving the promising charging performance. The 716 higher EUE confirms that more number of nodes are getting 717 charged in time. It is observed from Figs. 10a, 10b, and 10c 718 that the EUE of FLCSD is relatively high with that of other 719 
TABLE 8

Simulation Parameters

\begin{tabular}{lc}
\hline Parameter & Value \\
\hline Network region & $100 \times 100 \mathrm{~m}^{2}$ \\
Number of nodes $(N)$ & $400-1000$ \\
Number of MCs $(k)$ & 5 \\
Battery capacity of nodes $\left(E_{\text {max }}\right)$ & $10.8 \mathrm{KJ}$ \\
Battery capacity of MCs $\left(E_{\text {mc }}\right)$ & $4000 \mathrm{KJ}$ \\
Moving energy consumption rate $\left(E_{\text {move }}\right)$ & $600 \mathrm{~J} / \mathrm{m}$ \\
Moving speed of MC $\left(v_{m c}\right)$ & $1-7 \mathrm{~m} / \mathrm{s}$ \\
Charging range $\left(C_{r}\right)$ & $2.7 \mathrm{~m}$ \\
Charging rate of MCs $\left(c_{\text {rate }}\right)$ & $5 \mathrm{~J} / \mathrm{s}$ \\
Charging energy efficiency rate $(\eta)$ & 0.68 \\
Simulation time & 200 hours \\
\hline
\end{tabular}

two schemes, with increasing number of nodes, simulation time, and moving speed of the $\mathrm{MC}$, respectively. This demonstrates the high performance of proposed scheme as compared to other ones. This can be understood with the fact that fuzzy logic in the FLCSD tends to make wise and efficient scheduling decisions which provides charging opportunity to more number of nodes in time. Thus, it maximizes the amount of energy transferred to the nodes. On the other hand, unlike FLCSD, in UCMC and OMC_TSP, the charging load of MCs is not efficiently balanced and thus they fail to cope with the heterogeneous and dynamic energy consumption of the nodes. Moreover, the efficient selection criteria for choosing the most suitable node for recharging is also missing in these schemes, as a result, lesser nodes get recharged in time, thereby reducing the EUE. Further, we can observe from Figs. 10a, 10b, and 10c that the EUE of all the schemes shows increasing trend while the value of corresponding network parameters is also increasing. This is reasonable because more number of charging requests are delivered to the MCs with the increased number of nodes (see Fig. 10a). 739 This ensures that more energy is obtained by the nodes while 740 satisfying the requests. On an average, the EUE of the FLCSD 741 is 7 and 12 percent higher than the UCMC and the 742 OMC_TSP, respectively. Similarly, as the simulation time 743 goes on (in Fig. 10b) and speed of the MC increases (in 744 Fig. 10c), more number of charging requests are eventually 745 served by the MCs, thereby increasing the overall obtained 746 energy by the nodes and so EUE.

\subsubsection{Survival Rate}

Survival rate (SR) is defined as the ratio of the number of suc- 749 cessfully charged nodes by the MCs and the total number of 750 requesting nodes. The comparison results for SR with vary- 751 ing simulation time and moving speed of MC are shown in 752 Figs. 10d and 11a, respectively. A high SR in the network 753 guarantees the connectivity and stability of the network, 754 thus it is vital for improving the charging performance. $\quad 755$

The FLCSD performs scheduling of the nodes by blend- 756 ing multiple factors together so as to respond more requests. 757 Also due to the load balanced network partitioning, each 758 MC takes shorter time to complete the recharging tasks, 759 thus more nodes would get charged and so the SR increases. 760 It is clear from the Figs. $10 \mathrm{~d}$ and $11 \mathrm{a}$ that the SR in the 761 FLCSD is always higher than that of the UCMC and the 762 OMC_TSP. Furthermore, in Fig. 10d, with the simulation 763 going on, the SR gets decreased. The reason is that, the 764 increase in the simulation time leads to a bigger number of 765 charging requests which impose a heavier load on the MC 766 for fulfilling the charging requests. Therefore, more 767 exhausted nodes will appear. Furthermore, the survival 768 rates of compared schemes sharply decreases than that of 769 the proposed FLCSD scheme as observed in Fig. 10d. This is 770 because, the compared schemes ignore making scheduling 771

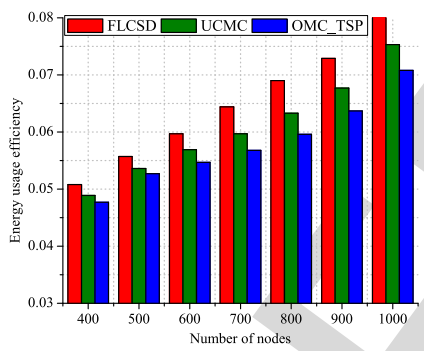

(a)

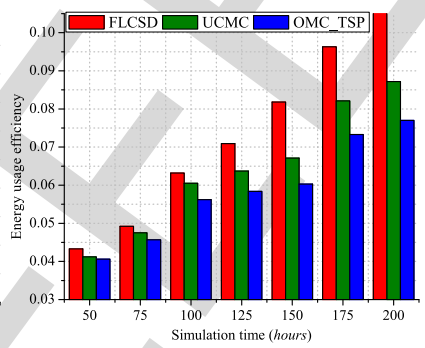

(b)

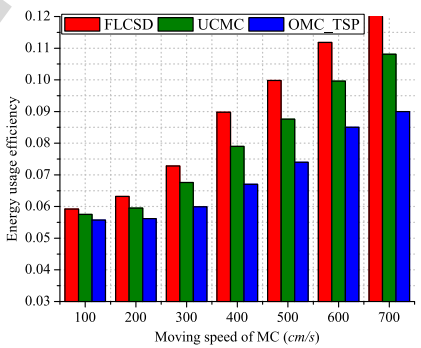

(c)

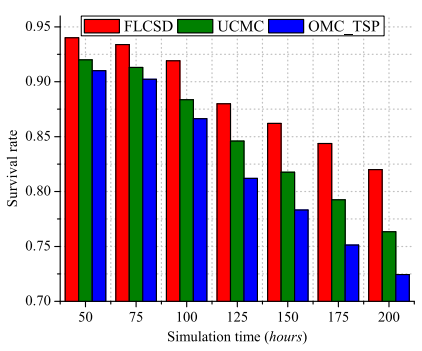

(d)

Fig. 10. Performance comparison of (a) EUE versus number of nodes. (b) EUE versus simulation time. (c) EUE versus moving speed of MC. (d) Survival rate versus simulation time.

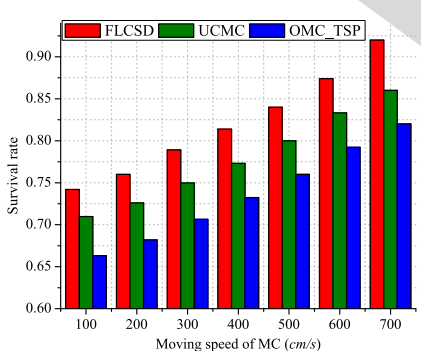

(a)

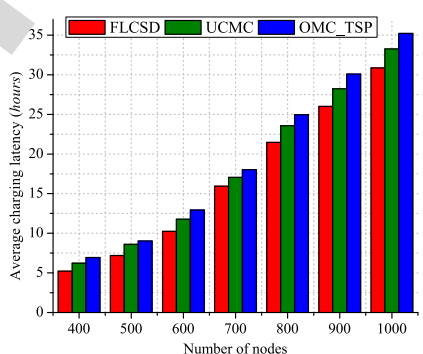

(b)

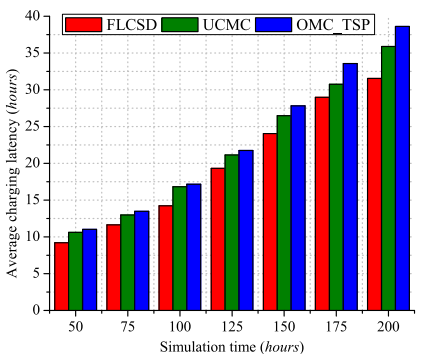

(c)

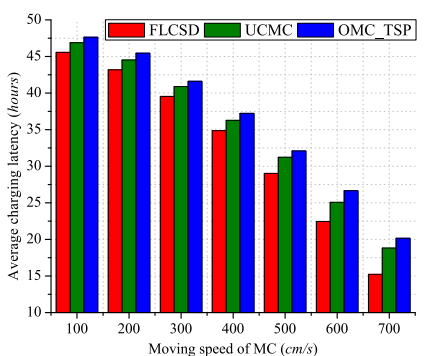

(d)

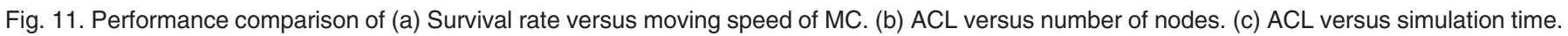

(d) ACL versus moving speed of MC. 
TABLE 9

Outcomes of ANOVA

\begin{tabular}{lccr}
\hline Performance metric & $F$-statistic & $p$-value & $F$-critical \\
\hline Energy usage efficiency & 12.51752712 & $3.11493 \mathrm{E}-05$ & 3.158842719 \\
Survival rate & 116.5302981 & $2.56652 \mathrm{E}-20$ & 3.168245967 \\
Average charging latency & 13.39063491 & $1.70901 \mathrm{E}-05$ & 3.158842719
\end{tabular}

decisions by incorporating multiple network attributes and consider fixed charging threshold for the nodes. This overlooks the exigency of recharging, making the nodes with more critical residual energy frequently die in case of large number of charging requests. Similarly, in Fig. 11a, the SR of all the simulated schemes increases when increasing moving speed of the MC. The rationale is that faster the MC moves, more requesting nodes the MC can serve. Hence, total number of successfully charged nodes increases, which makes the survival rate of all these schemes rise up.

\subsubsection{Average Charging Latency}

Average charging latency (ACL) is defined as the average time interval since the arrival time of a charging request to its completion time by the MC. The average charging latency is less, which means other critical nodes have to wait for a shorter time period, thereby preventing them to die earlier due to energy exhaustion.

It is clear to see in Figs. $11 \mathrm{~b}$ and 11c that the ACL of FLCSD scheme is always less than that of others with varying number of nodes and simulation time, respectively. The reason is clear as the MCs have to travel a lesser distance because a lot of sensors are charged through multi-node charging; second, requesting nodes are evenly distributed in the network by balancing their traffic load, thereby reducing the charging burden on the MCs and also shortening the response time for recharging the nodes. As a result, the MC tends to serve more number of requests while reducing the ACL in the network. Moreover, adaptive charging thresholds help nodes to send their charging requests in time without much delaying their charging response time. On the other hand, state-of-the-art schemes fail to consider above factors and make inefficient scheduling decisions. Moreover, charging workload of each MC remain unbalanced in the network and thus the ACL dramatically increases. Fig. 11d plots the ACL obtained by the simulated schemes for different moving speed of the MC. We can easily see that the ACL decreases as the speed of MC increases but the decreasing paces are different. This is due to the fact that if the speed of MC is higher, it takes less traveling time to serve the requesting nodes and this decreases the ACL. At the beginning, when moving speed of the $\mathrm{MC}$ are $\{1,2,3\} \mathrm{m} / \mathrm{s}$, the ACL of all the schemes decreases at the same rate. As the speed of MC further increases, the ACL of the FLCSD scheme decreases more rapidly. Moreover, the ACL of FLCSD is recorded the lowest, i.e., 6 percent lower than the UCMC and 9 percent lower than the OMC_TSP.

\subsubsection{Statistical Validation}

In this section, we check the statistical significance of the simulation results through hypothesis testing. We use analysis of variance (ANOVA) [34] to accomplish the same. ANOVA

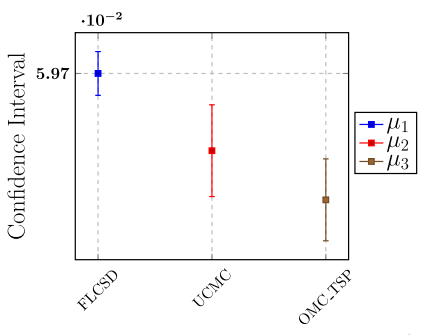

(a)

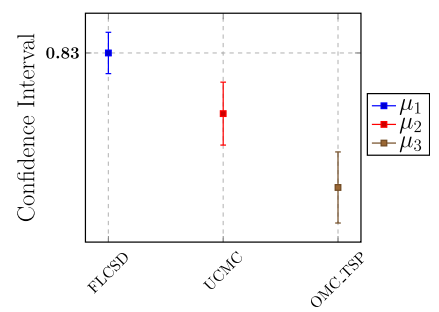

(b)

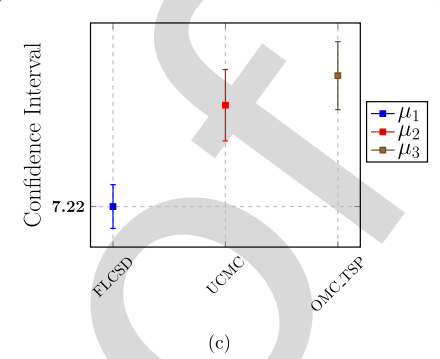

Fig. 12. Confidence interval plots for FLCSD, UCMC, and OMC_TSP schemes in terms of (a) EUE, (b) SR, and (c) ACL.

is a statistical method, mainly used to differentiate between 822 two or more means. More specifically, it helps to judge 823 whether a null hypothesis (" $H_{0}$ : the given groups have the 824 same means"), can be rejected. Let $F$-statistic be the out- 825 come of the ANOVA test. There are two important condi- 826 tions for rejecting a null hypothesis, one is $p$-value should 827 not be higher than the significance level $(\alpha)$ and the other is 828 $F$-statistic must be greater than $F$-critical. We have per- 829 formed the ANOVA test on each performance metric with 830 $\alpha=0.05$ by considering 20 samples of each scheme under 831 the same network instance. We define the null hypothesis as 832 " $H_{0}: \mu_{F L C S D}=\mu_{U C M C}=\mu_{O M C \_T S P}$ " and the alternative 833 hypothesis as " $H_{1}: \mu_{F L C S D} \neq \mu_{U C M C} \neq \mu_{O M O_{-} T S P}$ ". The out- 834 comes of ANOVA test in term of $F$-statistic, $p$-value, and 835 $F$-critical are presented in Table 9. On analyzing the results, 836 we can observe that both the aforementioned conditions are 837 satisfied (i.e., the $F$-statistic is greater than the $F$-critical 838 and $p$-value is much less than 0.05). Thus, we deduce that 839 the null hypothesis is rejected. This means that there are sta- 840 tistically significant differences in the means of each perfor- 841 mance metric for all the simulated schemes. We have also 842 shown the 95 percent confidence interval of the means of the 843 simulated schemes with respect to all the performance met- 844 rics. Fig. 12 depicts the interval plots using the normal distri- 845 bution, where $\mu_{1}, \mu_{2}$, and $\mu_{3}$ denote the mean of FLCSD, 846 UCMC, and OMC_TSP, respectively.

\section{Conclusion}

Taking into account the MC's limited power supply and 849 multi-node energy transfer capability, we have investigated 850 the charging scheduling problem, using multiple MCs in 851 on-demand WRSNs. First, we have presented a novel net- 852 work partitioning approach to distribute the MCs in a load 853 balanced manner. Next, with an objective of avoiding the 854 unfair charging response by the MCs, we have established a 855 mathematical formulation to calculate the adaptive recharg- 856 ing thresholds for the nodes. Finally, we have adopted the 857 Mamdani Fuzzy Inference System which determines the 858 charging schedules of the nodes by wisely integrating 859 
multiple attributes, such as residual energy, distance to $\mathrm{MC}$, critical node density, and energy consumption rate. Rigorous simulations have been carried out to prove the supremacy of the proposed FLCSD scheme over the state-of-the-art schemes. The results have revealed that as compared to the state-of-the-art schemes, the FLCSD provides better stability by increasing the number of survived nodes and boosts the charging performance by achieving both low average charging latency and high energy usage efficiency. The simulation results are also validated through the statistical ANOVA test. However, the proposed scheme has not considered the coordination among the MCs for recharging the nodes as well as the MCs, which may further improve the charging performance, especially in large-scale WRSNs. We will try to incorporate this feature in our future work by exploring new multi-attribute decision making methods.

\section{References}

[1] M. Ayaz, M. Ammad-uddin, I. Baig, and E. M. Aggoune, "Wireless sensor's civil applications, prototypes, and future integration possibilities: A review," IEEE Sensors J., vol. 18, no. 1, pp. 4-30, Jan. 2018.

[2] P. Kar and S. Misra, "Reliable and efficient data acquisition in wireless sensor networks in the presence of transfaulty nodes," IEEE Trans. Netw. Service Manag., vol. 13, no. 1, pp. 99-112, Mar. 2016.

[3] S. Gao, H. Zhang, and S. K. Das, "Efficient data collection in wireless sensor networks with path-constrained mobile sinks," IEEE Trans. Mobile Comput., vol. 10, no. 4, pp. 592-608, Apr. 2011.

[4] B. Fateh and M. Govindarasu, "Joint scheduling of tasks and messages for energy minimization in interference-aware real-time sensor networks," IEEE Trans. Mobile Comput., vol. 14, no. 1, pp. 86-98, Jan. 2015.

[5] S. Guo, Y. Shi, Y. Yang, and B. Xiao, "Energy efficiency maximization in mobile wireless energy harvesting sensor networks," IEEE Trans. Mobile Comput., vol. 17, no. 7, pp. 1524-1537, Jul. 2018.

[6] A. Mehrabi and K. Kim, "General framework for network throughput maximization in sink-based energy harvesting wireless sensor networks," IEEE Trans. Mobile Comput., vol. 16, no. 7, pp. 1881-1896, Jul. 2017.

[7] A. Kurs, A. Karalis, R. Moffatt, J. D. Joannopoulos, P. Fisher, and M. Soljačić, "Wireless power transfer via strongly coupled magnetic resonances," Science, vol. 317, no. 5834, pp. 83-86, 2007.

[8] K. Kang, Y. S. Meng, J. Bréger, C. P. Grey, and G. Ceder, "Electrodes with high power and high capacity for rechargeable lithium batteries," Science, vol. 311, no. 5763, pp. 977-980, 2006.

[9] M. Hu, Z. Chen, K. Peng, X. Ma, P. Zhou, and J. Liu, "Periodic charging for wireless sensor networks with multiple portable chargers," IEEE Access, vol. 7, pp. 2612-2623, 2018.

[10] L. Xie, Y. Shi, Y. T. Hou, W. Lou, H. D. Sherali, and S. F. Midkiff, "Multi-node wireless energy charging in sensor networks," IEEE/ ACM Trans. Netw., vol. 23, no. 2, pp. 437-450, Apr. 2015.

[11] A. Kaswan, A. Tomar, and P. K. Jana, "An efficient scheduling scheme for mobile charger in on-demand wireless rechargeable sensor networks," J. Netw. Comput. Appl., vol. 114, pp. 123-134, 2018.

[12] L. He, L. Kong, Y. Gu, J. Pan, and T. Zhu, "Evaluating the ondemand mobile charging in wireless sensor networks," IEEE Trans. Mobile Comput., vol. 14, no. 9, pp. 1861-1875, Sep. 2015.

[13] C. Lin, J. Zhou, C. Guo, H. Song, G. Wu, and M. S. Obaidat, "TSCA: A temporal-spatial real-time charging scheduling algorithm for on-demand architecture in wireless rechargeable sensor networks," IEEE Trans. Mobile Comput., vol. 17, no. 1, pp. 211-224, Jan. 2018.

[14] C. Lin, Z. Wang, D. Han, Y. Wu, C. W. Yu, and G. Wu, “TADP: Enabling temporal and distantial priority scheduling for ondemand charging architecture in wireless rechargeable sensor networks," J. Syst. Archit., vol. 70, pp. 26-38, 2016.

[15] Y. Shu et al., "Near-optimal velocity control for mobile charging in wireless rechargeable sensor networks," IEEE Trans. Mobile Comput., vol. 15, no. 7, pp. 1699-1713, Jul. 2016.
[16] L. Fu, P. Cheng, Y. Gu, J. Chen, and T. He, “Optimal charging in 928 wireless rechargeable sensor networks," IEEE Trans. Veh. Technol., 929 vol. 65, no. 1, pp. 278-291, Jan. 2016.

[17] A. Kurs, R. Moffatt, and M. Soljačić, "Simultaneous mid-range 931 power transfer to multiple devices," Appl. Phys. Lett., vol. 96, no. 4, 932 2010, Art. no. 044102.

[18] W. Xu, W. Liang, H. Kan, Y. Xu, and X. Zhang, "Minimizing the 934 longest charge delay of multiple mobile chargers for wireless 935 rechargeable sensor networks by charging multiple sensors simul- 936 taneously," in Proc. IEEE 39th Int. Conf. Distrib. Comput. Syst., 2019, 937 pp. 881-890.

[19] W. Xu, W. Liang, X. Jia, H. Kan, Y. Xu, and X. Zhang, “Minimizing 939 the maximum charging delay of multiple mobile chargers under 940 the multi-node energy charging scheme," IEEE Trans. Mobile Com- 941 put., to be published, doi: 10.1109/TMC.2020.2973979. 942

[20] G. Han, H. Guan, J. Wu, S. Chan, L. Shu, and W. Zhang, “An 943 uneven cluster-based mobile charging algorithm for wireless 944 rechargeable sensor networks," IEEE Syst. J., vol. 13, no. 4, 945 pp. 3747-3758, Dec. 2019.

[21] T. Rault, "Avoiding radiation of on-demand multi-node energy 947 charging with multiple mobile chargers," Comput. Commun., vol. 134, 948 pp. 42-51, 2019.

[22] R. V. Kulkarni, A. Forster, and G. K. Venayagamoorthy, 950 "Computational intelligence in wireless sensor networks: A 951 survey," IEEE Commun. Surveys Tuts., vol. 13, no. 1, pp. 68-96, 952 First Quarter 2011.

[23] L. Jiang, X. Wu, G. Chen, and Y. Li, "Effective on-demand mobile 954 charger scheduling for maximizing coverage in wireless recharge- 955 able sensor networks," Mobile Netw. Appl., vol. 19, no. 4, pp. 543-551, 956 2014.

[24] W. Liang, W. Xu, X. Ren, X. Jia, and X. Lin, "Maintaining large- 958 scale rechargeable sensor networks perpetually via multiple 959 mobile charging vehicles," ACM Trans. Sensor Netw., vol. 12, no. 2, 960 2016, Art. no. 14.

[25] C. Lin, D. Han, J. Deng, and G. Wu, “P ${ }^{2}$ S: A primary and passer- 962 by scheduling algorithm for on-demand charging architecture in 963 wireless rechargeable sensor networks," IEEE Trans. Veh. Technol., 964 vol. 66, no. 9, pp. 8047-8058, Sep. 2017.

[26] W. Xu, W. Liang, X. Lin, and G. Mao, "Efficient scheduling of mul- 966 tiple mobile chargers for wireless sensor networks," IEEE Trans. 967 Veh. Technol., vol. 65, no. 9, pp. 7670-7683, Sep. 2016.

[27] Y. Ma, W. Liang, and W. Xu, "Charging utility maximization in 969 wireless rechargeable sensor networks by charging multiple sen- 970 sors simultaneously," IEEE/ACM Trans. Netw., vol. 26, no. 4, 971 pp. 1591-1604, Aug. 2018.

[28] N. Gharaei, Y. D. Al-Otaibi, S. A. Butt, S. J. Malebary, S. Rahim, 973 and G. Sahar, "Energy-efficient tour optimization of wireless 974 mobile chargers for rechargeable sensor networks," IEEE Syst. J., 975 to be published, doi: 10.1109/JSYST.2020.2968968. 976

[29] L. Mo, A. Kritikakou, and S. He, "Energy-aware multiple mobile 977 chargers coordination for wireless rechargeable sensor networks," 978 IEEE Internet Things J., vol. 6, no. 5, pp. 8202-8214, Oct. 2019.

[30] L. Khelladi, D. Djenouri, M. Rossi, and N. Badache, "Efficient on- 980 demand multi-node charging techniques for wireless sensor 981 networks," Comput. Commun., vol. 101, pp. 44-56, 2017.

[31] A. Tomar, L. Muduli, and P. K. Jana, "An efficient scheduling scheme 983 for on-demand mobile charging in wireless rechargeable sensor 984 networks," Pervasive Mobile Comput., vol. 59, 2019, Art. no. 101074. 985

[32] J. Zhu, Y. Feng, M. Liu, G. Chen, and Y. Huang, "Adaptive online 986 mobile charging for node failure avoidance in wireless recharge- 987 able sensor networks," Comput. Commun., vol. 126, pp. 28-37, 988 2018.

[33] C. Lin et al., "GTCCS: A game theoretical collaborative charging 990 scheduling for on-demand charging architecture," IEEE Trans. 991 Veh. Technol., vol. 67, no. 12, pp. 12 124-12 136, Dec. 2018.

[34] K. E. Muller and B. A. Fetterman, Regression and ANOVA: An 993 Integrated Approach Using SAS Software. Cary, NC, USA: SAS 994 Institute, 2003.

$\triangleright$ For more information on this or any other computing topic, 996 please visit our Digital Library at www.computer.org/csdl. 997 